FEDERAL RESERVE BANK OF SAN FRANCISCO

WORKING PAPER SERIES

\title{
Modeling Yields at the Zero Lower Bound: Are Shadow Rates the Solution?
}

\author{
Jens H. E. Christensen, \\ Federal Reserve Bank of San Francisco \\ Glenn D. Rudebusch, \\ Federal Reserve Bank of San Francisco
}

December 2013

Working Paper 2013-39

http://www.frbsf.org/publications/economics/papers/2013/wp2013-39.pdf

The views in this paper are solely the responsibility of the authors and should not be interpreted as reflecting the views of the Federal Reserve Bank of San Francisco or the Board of Governors of the Federal Reserve System. 


\title{
Modeling Yields at the Zero Lower Bound: Are Shadow Rates the Solution?
}

\author{
Jens H. E. Christensen \\ and \\ Glenn D. Rudebusch \\ Federal Reserve Bank of San Francisco \\ 101 Market Street, Mailstop 1130 \\ San Francisco, CA 94105 \\ |
}

\begin{abstract}
Recent U.S. Treasury yields have been constrained to some extent by the zero lower bound (ZLB) on nominal interest rates. In modeling these yields, we compare the performance of a standard affine Gaussian dynamic term structure model (DTSM), which ignores the ZLB, and a shadowrate DTSM, which respects the ZLB. We find that the standard affine model is likely to exhibit declines in fit and forecast performance with very low interest rates. In contrast, the shadow-rate model mitigates ZLB problems significantly and we document superior performance for this model class in the most recent period.
\end{abstract}

JEL Classification: G12, E43, E52, E58.

Keywords: term structure modeling, zero lower bound, monetary policy.

We thank conference participants at the FRBSF Workshop on "Term Structure Modeling at the Zero Lower Bound"-especially Don Kim-for helpful comments. The views in this paper are solely the responsibility of the authors and should not be interpreted as reflecting the views of the Federal Reserve Bank of San Francisco or the Board of Governors of the Federal Reserve System. We thank Lauren Ford for excellent research assistance.

This version: December 17, 2013. 


\section{Introduction}

With nominal yields on government debt in several countries having fallen very near their zero lower bound (ZLB), understanding how to model the term structure of interest rates when some of those interest rates are near the ZLB is an issue that commands attention both for bond portfolio pricing and risk management and for macroeconomic and monetary policy analysis. The timing of the ZLB period in the United States can be seen in Figure 11 The start of the ZLB period is commonly dated to December 16, 2008, when the Federal Open Market Committee (FOMC) lowered its target policy rate - the overnight federal funds rate - to a range of 0 to $1 / 4$ percent.

The key empirical question of the paper is to extract reliable market-based measures of expectations for future monetary policy when nominal interest rates are near the ZLB. Unfortunately, the workhorse representation in finance for bond pricing - the affine Gaussian dynamic term structure model - ignores the ZLB and places positive probabilities on negative interest rates as we will show. This counterfactual outcome results from ignoring the existence of a readily available currency for transactions. For in the real world, an investor always has the option of holding cash, and the zero nominal yield of cash will dominate any security with a negative yield 2 Instead, to handle the problem of near-zero yields, we rely on the shadow-rate arbitrage-free Nelson-Siegel (AFNS) model class introduced in Christensen and Rudebusch (2013) 3 These are latent-factor models where the state variables have standard Gaussian dynamics, but the short rate is given an interpretation of a shadow rate in the spirit of Black (1995) to account for the effect on bond pricing from the existence of the option to hold currency. As a consequence, the models respect the ZLB. Furthermore, due to the Gaussian dynamics, these shadow-rate models are as flexible and empirically tractable as regular AFNS models.

In the empirical analysis, we compare the results from this new shadow-rate AFNS model to those obtained from a regular AFNS model estimated on the same sample. We find that shadow-rate models can provide better fit as measured by in-sample metrics such as the RMSEs of fitted yields and the likelihood values. Still, it is evident from these in-sample results that a standard three-factor Gaussian dynamic term structure model like our Gaussian three-factor AFNS model has enough flexibility to fit the cross-section of yields fairly well at each point in time even when the shorter end of the yield curve is flattened out at the ZLB. However, it is not the case that the Gaussian model can account for all aspects of the term structure at the ZLB. Indeed, we show that our estimated three-factor Gaussian model clearly fails along two dimensions. First, despite fitting the yield curve,

\footnotetext{
${ }^{1}$ The data are nominal U.S. Treasury zero-coupon yields and described later in the paper.

${ }^{2}$ Actually, the ZLB can be a somewhat soft floor. The non-negligible costs of transacting in and holding large amounts of currency have allowed government bond yields to push slightly below zero in a few countries, notably in Denmark recently. To capture a lower bound on bond yields that depends on institutional frictions, we could replace the lower bound of zero with some appropriate, possibly time-varying, negative epsilon.

${ }^{3}$ See Diebold and Rudebusch (2013) for a comprehensive presentation of related applications of the AFNS model.
} 


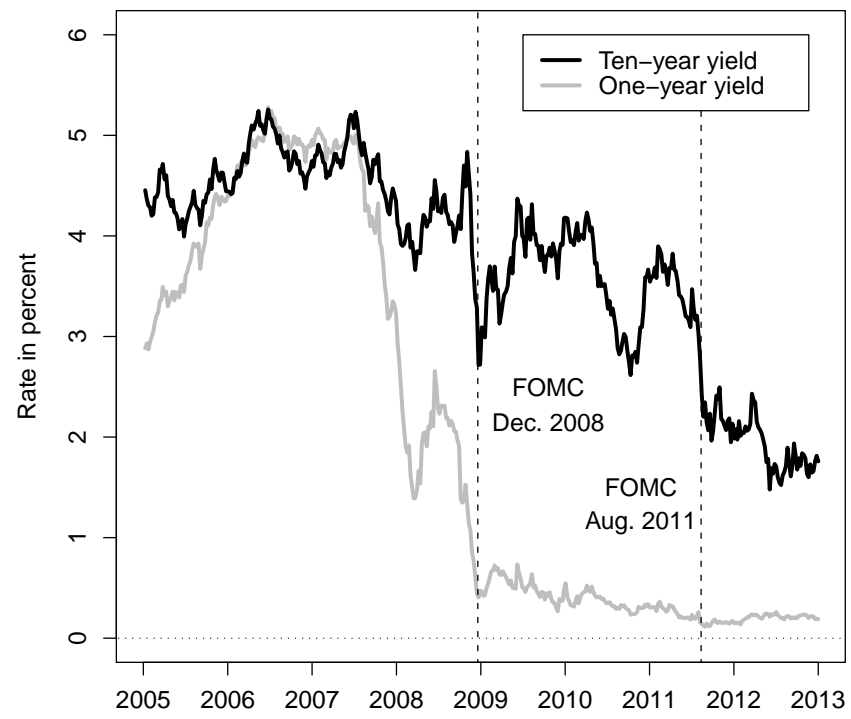

Figure 1: Treasury Yields Since 2005.

One- and ten-year weekly U.S. Treasury zero-coupon bond yields from January 7, 2005, to December 28, 2012.

the model cannot capture the dynamics of yields at the ZLB. One stark indication of this is the high probability the model assigns to negative future short rates - obviously a poor prediction. Second, the standard model misses the compression of yield volatility that occurs at the ZLB as expected future short rates are pinned near zero, longer-term rates fluctuate less. The shadow-rate model, even without incorporating stochastic volatility, can capture this effect. In terms of forecasting future short rates, we first establish that the regular AFNS model is competitive over the normal period from 1995 to 2008. Thus, this model could have been expected to continue to perform well in the most recent period, if only it had not been for the problems associated with the ZLB. Second, we show that during the most recent period the shadow-rate model stands out in terms of forecasting future short rates in addition to performing on par with the regular model during the normal period. Third, the deterioration in short rate forecasts implies that the regular model delivers exaggerated estimates of the policy expectations embedded in the yield curve in recent years. In turn, this leads us to conclude that its term premium estimates are artificially low during that period 4 As a consequence of these findings combined, we recommend to use a shadow-rate modeling approach not only when yields are as low as they were towards the end of our sample, but in general.

\footnotetext{
${ }^{4}$ Ichiue and Ueno (2013) also compare standard and shadow-rate Gaussian models for U.S. Treasury data and find deterioration in the performance of their standard model during the most recent period. However, they only study two-factor models.
} 
Finally, we should mention two alternative frameworks to modeling yields near the ZLB that guarantee positive interest rates: stochastic-volatility models with square-root processes and Gaussian quadratic models. Both of these approaches suffer from the theoretical weakness that they treat the ZLB as a reflecting barrier and not as an absorbing one as in the shadow-rate model. Empirically, of course, the recent prolonged period of very low interest rates seem more consistent with an absorbing state. In addition, Dai and Singleton (2002) disparage the fit of stochastic-volatility models, while Kim and Singleton (2012) compare quadratic and shadow-rate empirical representations and find a slight preference for the latter. Still, we consider all three modeling approaches to be worthy of further investigation, but we view the shadow-rate model to be of particular interest because away from the ZLB it reduces exactly to the standard Gaussian affine model, which is by far the most popular dynamic term structure model. Therefore, the entire voluminous literature on affine Gaussian models remains completely applicable and relevant when given a modest shadow-rate tweak to handle the ZLB.

The rest of the paper is structured as follows. Section 2 describes Gaussian models in general as well as a specific empirical Gaussian model that we consider, while Section 3 details our shadow-rate model. Section 4 contains our empirical findings and discusses the implications for assessing policy expectations and term premiums in the current low-yield environment. Section 5 concludes. Two appendices contain additional technical details.

\section{A Standard Gaussian Term Structure Model}

In this section, we provide an overview of the affine Gaussian term structure model, which ignores the ZLB, and describe an empirical example of this model.

\subsection{The General Model}

Let $P_{t}(\tau)$ be the price of a zero-coupon bond at time $t$ that pays $\$ 1$, at maturity $t+\tau$. Under standard assumptions, this price is given by

$$
P_{t}(\tau)=E_{t}^{P}\left[\frac{M_{t+\tau}}{M_{t}}\right]
$$

where the stochastic discount factor, $M_{t}$, denotes the value at time $t_{0}$ of a claim at a future date $t$, and the superscript $P$ refers to the actual, or real-world, probability measure underlying the dynamics of $M_{t}$. (As we will discuss in the next section, there is no restriction in this standard setting to constrain $P_{t}(\tau)$ from rising above its par value; that is, the ZLB is ignored.)

We follow the usual reduced-form empirical finance approach that models bond prices with unobservable (or latent) factors, here denoted as $X_{t}$, and the assumption of no residual arbitrage 
opportunities. We assume that $X_{t}$ follows an affine Gaussian process with constant volatility, with dynamics in continuous time given by the solution to the following stochastic differential equation $(\mathrm{SDE})$ :

$$
d X_{t}=K^{P}\left(\theta^{P}-X_{t}\right) d t+\Sigma d W_{t}^{P}
$$

where $K^{P}$ is an $n \times n$ mean-reversion matrix, $\theta^{P}$ is an $n \times 1$ vector of mean levels, $\Sigma$ is an $n \times n$ volatility matrix, and $W_{t}^{P}$ is an $n$-dimensional Brownian motion. The dynamics of the stochastic discount function are given by

$$
d M_{t}=r_{t} M_{t} d t+\Gamma_{t}^{\prime} M_{t} d W_{t}^{P}
$$

and the instantaneous risk-free rate, $r_{t}$, is assumed affine in the state variables

$$
r_{t}=\delta_{0}+\delta_{1}^{\prime} X_{t}
$$

where $\delta_{0} \in R$ and $\delta_{1} \in R^{n}$. The risk premiums, $\Gamma_{t}$, are also affine

$$
\Gamma_{t}=\gamma_{0}+\gamma_{1} X_{t}
$$

where $\gamma_{0} \in R^{n}$ and $\gamma_{1} \in R^{n \times n}$.

Duffie and Kan (1996) show that these assumptions imply that zero-coupon yields are also affine in $X_{t}$ :

$$
y_{t}(\tau)=-\frac{1}{\tau} A(\tau)-\frac{1}{\tau} B(\tau)^{\prime} X_{t}
$$

where $A(\tau)$ and $B(\tau)$ are given as solutions to the following system of ordinary differential equations

$$
\begin{aligned}
& \frac{d B(\tau)}{d \tau}=-\delta_{1}-\left(K^{P}+\Sigma \gamma_{1}\right)^{\prime} B(\tau), \quad B(0)=0 \\
& \frac{d A(\tau)}{d \tau}=-\delta_{0}+B(\tau)^{\prime}\left(K^{P} \theta^{P}-\Sigma \gamma_{0}\right)+\frac{1}{2} \sum_{j=1}^{n}\left[\Sigma^{\prime} B(\tau) B(\tau)^{\prime} \Sigma\right]_{j, j}, \quad A(0)=0 .
\end{aligned}
$$

Thus, the $A(\tau)$ and $B(\tau)$ functions are calculated as if the dynamics of the state variables had a constant drift term equal to $K^{P} \theta^{P}-\Sigma \gamma_{0}$ instead of the actual $K^{P} \theta^{P}$ and a mean-reversion matrix equal to $K^{P}+\Sigma \gamma_{1}$ as opposed to the actual $K^{P}$. The probability measure with these alternative dynamics is frequently referred to as the risk-neutral, or $Q$, probability measure since the expected return on any asset under this measure is equal to the risk-free rate $r_{t}$ that a risk-neutral investor would demand. The difference is determined by the risk premium $\Gamma_{t}$ and reflects investors' aversion to the risks embodied in $X_{t}$. 
Finally, we define the term premium as

$$
T P_{t}(\tau)=y_{t}(\tau)-\frac{1}{\tau} \int_{t}^{t+\tau} E_{t}^{P}\left[r_{s}\right] d s
$$

That is, the term premium is the difference in expected return between a buy and hold strategy for a $\tau$-year Treasury bond and an instantaneous rollover strategy at the risk-free rate $r_{t}$.

\subsection{An Empirical Affine Model}

A wide variety of Gaussian term structure models have been estimated. Here, we describe an empirical representation from the literature that uses high-frequency observations on U.S. yields from a sample that includes the recent ZLB period. It improves the econometric identification of the latent factors, which facilitates model estimation 5 The Gaussian term structure model we consider is an update of the one used by Christensen and Rudebusch (2012). This "CR model" is an arbitrage-free Nelson-Siegel (AFNS) representation with three latent state variables, $X_{t}=\left(L_{t}, S_{t}, C_{t}\right)$. These are described by the following system of SDEs under the risk-neutral $Q$-measure 6

$$
\left(\begin{array}{c}
d L_{t} \\
d S_{t} \\
d C_{t}
\end{array}\right)=\left(\begin{array}{ccc}
0 & 0 & 0 \\
0 & \lambda & -\lambda \\
0 & 0 & \lambda
\end{array}\right)\left[\left(\begin{array}{c}
\theta_{1}^{Q} \\
\theta_{2}^{Q} \\
\theta_{3}^{Q}
\end{array}\right)-\left(\begin{array}{c}
L_{t} \\
S_{t} \\
C_{t}
\end{array}\right)\right] d t+\Sigma\left(\begin{array}{c}
d W_{t}^{L, Q} \\
d W_{t}^{S, Q} \\
d W_{t}^{C, Q}
\end{array}\right), \quad \lambda>0
$$

where $\Sigma$ is the constant covariance (or volatility) matrix.

In addition, the instantaneous risk-free rate is defined by

$$
r_{t}=L_{t}+S_{t}
$$

This specification implies that zero-coupon bond yields are given by

$$
y_{t}(\tau)=L_{t}+\left(\frac{1-e^{-\lambda \tau}}{\lambda \tau}\right) S_{t}+\left(\frac{1-e^{-\lambda \tau}}{\lambda \tau}-e^{-\lambda \tau}\right) C_{t}-\frac{A(\tau)}{\tau}
$$

where the factor loadings in the yield function match the level, slope, and curvature loadings introduced in Nelson and Siegel (1987). The final yield-adjustment term, $A(\tau) / \tau$, captures convexity effects due to Jensen's inequality.

The model is completed with a risk premium specification that connects the factor dynamics to

\footnotetext{
${ }^{5}$ Difficulties in estimating Gaussian term structure models are discussed in Christensen et al. (2011), who propose using a Nelson-Siegel structure to avoid them.

${ }^{6}$ Two details regarding this specification are discussed in Christensen et al. (2011). First, with a unit root in the level factor under the pricing measure, the model is not arbitrage-free with an unbounded horizon; therefore, as is often done in theoretical discussions, we impose an arbitrary maximum horizon. Second, we identify this class of models by fixing the $\theta^{Q}$ means under the $Q$-measure at zero without loss of generality.
} 
the dynamics under the real-world $P$-measure 7 The maximally flexible specification of the AFNS model has $P$-dynamics given by 8

$$
\left(\begin{array}{c}
d L_{t} \\
d S_{t} \\
d C_{t}
\end{array}\right)=\left(\begin{array}{ccc}
\kappa_{11}^{P} & \kappa_{12}^{P} & \kappa_{13}^{P} \\
\kappa_{21}^{P} & \kappa_{22}^{P} & \kappa_{23}^{P} \\
\kappa_{31}^{P} & \kappa_{32}^{P} & \kappa_{33}^{P}
\end{array}\right)\left[\left(\begin{array}{c}
\theta_{1}^{P} \\
\theta_{2}^{P} \\
\theta_{3}^{P}
\end{array}\right)-\left(\begin{array}{c}
L_{t} \\
S_{t} \\
C_{t}
\end{array}\right)\right] d t+\left(\begin{array}{ccc}
\sigma_{11} & 0 & 0 \\
\sigma_{21} & \sigma_{22} & 0 \\
\sigma_{31} & \sigma_{32} & \sigma_{33}
\end{array}\right)\left(\begin{array}{c}
d W_{t}^{L, P} \\
d W_{t}^{S, P} \\
d W_{t}^{C, P}
\end{array}\right) .
$$

Using both in- and out-of-sample performance measures, CR went through a careful empirical analysis to justify various zero-value restrictions on the $K^{P}$ matrix. Imposing these restrictions results in the following dynamic system for the $P$-dynamics:

$$
\left(\begin{array}{c}
d L_{t} \\
d S_{t} \\
d C_{t}
\end{array}\right)=\left(\begin{array}{ccc}
10^{-7} & 0 & 0 \\
\kappa_{21}^{P} & \kappa_{22}^{P} & \kappa_{23}^{P} \\
0 & 0 & \kappa_{33}^{P}
\end{array}\right)\left(\left(\begin{array}{c}
0 \\
\theta_{2}^{P} \\
\theta_{3}^{P}
\end{array}\right)-\left(\begin{array}{c}
L_{t} \\
S_{t} \\
C_{t}
\end{array}\right)\right) d t+\Sigma\left(\begin{array}{c}
d W_{t}^{L, P} \\
d W_{t}^{S, P} \\
d W_{t}^{C, P}
\end{array}\right)
$$

where the covariance matrix $\Sigma$ is assumed diagonal and constant. Note that in this specification, the Nelson-Siegel level factor is restricted to be an independent unit-root process under both probability measures 9 As discussed in $\mathrm{CR}$, this restriction helps improve forecast performance independent of the specification of the remaining elements of $K^{P}$. Because interest rates are highly persistent, empirical autoregressive models, including DTSMs, suffer from substantial small-sample estimation bias. Specifically, model estimates will generally be biased toward a dynamic system that displays much less persistence than the true process (so estimates of the real-world mean-reversion matrix, $K^{P}$, are upward biased). Furthermore, if the degree of interest rate persistence is underestimated, future short rates would be expected to revert to their mean too quickly causing their expected longer-term averages to be too stable. Therefore, the bias in the estimated dynamics distorts the decomposition of yields and contaminates estimates of long-maturity term premia. As described in detail in Bauer et al. (2012), bias-corrected $K^{P}$ estimates are typically very close to a unit-root process, so we view the imposition of the unit-root restriction as a simple shortcut to overcome small-sample estimation bias.

We re-estimated this CR model over a larger sample of weekly nominal U.S. Treasury zerocoupon yields from January 4, 1985, until December 28, 2012, for eight maturities: three months, six months, one year, two years, three years, five years, seven years, and ten years 10 The model

\footnotetext{
${ }^{7}$ It is important to note that there are no restrictions on the dynamic drift components under the empirical $P$ measure beyond the requirement of constant volatility. To facilitate empirical implementation, we use the essentially affine risk premium introduced in Duffee (2002).

${ }^{8}$ As noted in Christensen et al. (2011), the unconstrained AFNS model has a sign restriction and three parameters less than the standard canonical three-factor Gaussian DTSM.

${ }^{9}$ Due to the unit-root property of the first factor, we can arbitrarily fix its mean at $\theta_{1}^{P}=0$.

${ }^{10}$ The yield data include three- and six-month Treasury bill yields from the H.15 series from the Federal Reserve
} 


\begin{tabular}{|c|ccc||c||c|c|}
\hline$K^{P}$ & $K_{\cdot, 1}^{P}$ & $K_{\cdot, 2}^{P}$ & $K_{\cdot, 3}^{P}$ & $\theta^{P}$ & & $\Sigma$ \\
\hline$K_{1, \cdot}^{P}$ & $10^{-7}$ & 0 & 0 & 0 & $\sigma_{11}$ & 0.0065 \\
& & & & & & $(0.0001)$ \\
$K_{2, \cdot}^{P}$ & 0.3753 & 0.4073 & -0.4277 & 0.0319 & $\sigma_{22}$ & 0.0100 \\
& $(0.1319)$ & $(0.1171)$ & $(0.0857)$ & $(0.0270)$ & & $(0.0002)$ \\
$K_{3, \cdot}^{P}$ & 0 & 0 & 0.6371 & -0.0237 & $\sigma_{33}$ & 0.0272 \\
& & & $(0.1607)$ & $(0.0073)$ & & $(0.0004)$ \\
\hline
\end{tabular}

\section{Table 1: Parameter Estimates for the CR Model.}

The estimated parameters of the $K^{P}$ matrix, $\theta^{P}$ vector, and diagonal $\Sigma$ matrix are shown for the CR model. The estimated value of $\lambda$ is $0.4455(0.0023)$. The numbers in parentheses are the estimated parameter standard deviations. The maximum log likelihood value is $66,388.06$.

parameter estimates are reported in Table 1. As in CR, we tested the significance of the four parameter restrictions imposed on $K^{P}$ in the CR model relative to the unrestricted AFNS model 11 As before, we found that the four parameter restrictions are not rejected by the data; thus, the CR model appears flexible enough to capture the relevant information in the data compared with an unrestricted model.

\subsection{Negative Short-Rate Projections in Standard Models}

Before turning to the description of the shadow rate model, it is useful to reinforce the basic motivation for our analysis by examining short rate forecasts from the estimated CR model. With regard to short rate forecasts, any standard affine Gaussian dynamic term structure model may place positive probabilities on future negative interest rates. Accordingly, Figure 2 shows the probability obtained from the CR model that the short rate three months out will be negative. Prior to 2008 the probabilities of future negative interest rates are negligible except for a brief period in 2003 and 2004 when the Fed's policy rate temporarily stood at one percent. However, near the ZLB - since late 2008 - the model is typically predicting substantial likelihoods of impossible realizations.

\section{A Shadow-Rate Model}

In this section, we describe an option-based approach to the shadow-rate model and estimate a shadow-rate analog to the CR model with U.S. data.

Board as well as off-the-run Treasury zero-coupon yields for the remaining maturities from the Gürkaynak et al. (2007) database, which is available at http://www.federalreserve.gov/pubs/feds/2006/200628/200628abs.html.

${ }^{11}$ That is, a test of the joint hypothesis $\kappa_{12}^{P}=\kappa_{13}^{P}=\kappa_{31}^{P}=\kappa_{32}^{P}=0$ using a standard likelihood ratio test. 


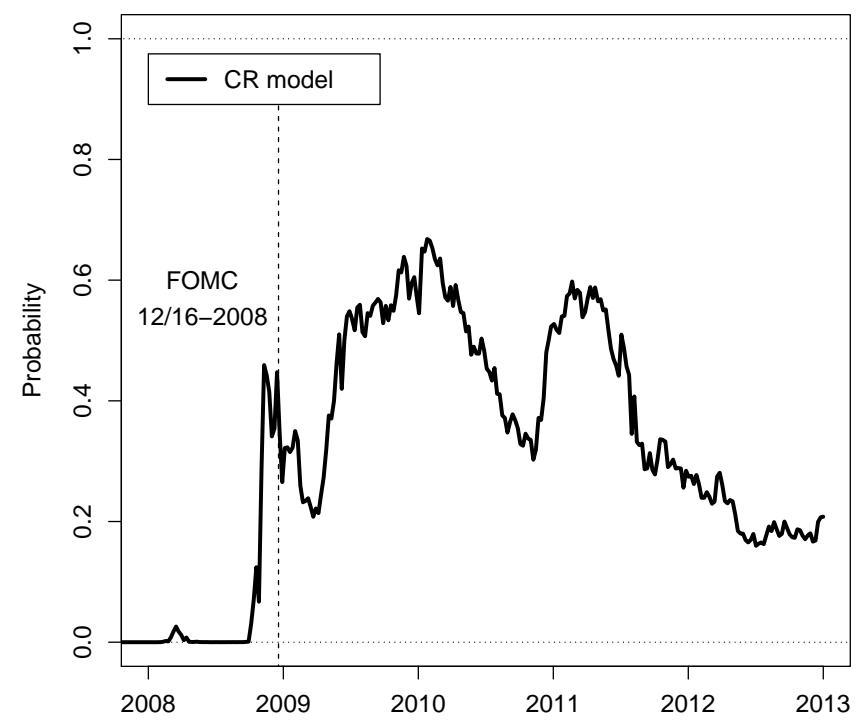

Figure 2: Probability of Negative Short Rates Since 2008.

Illustration of the conditional probability of negative short rates three months ahead from the CR model.

\subsection{The Option-Based Approach to the Shadow-Rate Model}

The concept of a shadow interest rate as a modeling tool to account for the ZLB can be attributed to Black (1995). He noted that the observed nominal short rate will be nonnegative because currency is a readily available asset to investors that carries a nominal interest rate of zero. Therefore, the existence of currency sets a zero lower bound on yields. To account for this ZLB, Black postulated as a modeling tool a shadow short rate, $s_{t}$, that is unconstrained by the ZLB. The usual observed instantaneous risk-free rate, $r_{t}$, which is used for discounting cash flows when valuing securities, is then given by the greater of the shadow rate or zero:

$$
r_{t}=\max \left\{0, s_{t}\right\}
$$

Accordingly, as $s_{t}$ falls below zero, the observed $r_{t}$ simply remains at the zero bound.

While Black (1995) described circumstances under which the zero bound on nominal yields might be relevant, he did not provide specifics for implementation. Gorovoi and Linetsky (2004) derive bond price formulas for the case of one-factor Gaussian and square-root shadow-rate models 12 Unfortunately, their results do not extend to multidimensional models. Instead, the small set of previous

\footnotetext{
${ }^{12}$ Ueno, Baba, and Sakurai (2006) use these formulas when calibrating a one-factor Gaussian model to a sample of Japanese government bond yields.
} 
research on shadow-rate models has relied on numerical methods for pricing 13 However, in light of the computational burden of these methods, there have been only two previous estimations of multifactor shadow-rate models: Ichiue and Ueno (2007) and Kim and Singleton (2012). Both of these studies undertake a full maximum likelihood estimation of their two-factor Gaussian shadow-rate models on Japanese bond yield data using the extended Kalman filter and numerical optimization.

To overcome the curse of dimensionality that limits numerical-based estimation of shadow-rate models, Krippner (2013) suggested an alternative option-based approach that makes shadow-rate models almost as easy to estimate as the corresponding non-shadow-rate model. To illustrate this approach, consider two bond-pricing situations: one without currency as an alternative asset and the other that has a currency in circulation that has a constant nominal value and no transaction costs. In the world without currency, the price of a shadow-rate zero-coupon bond, $P_{t}(\tau)$, may trade above par, that is, its risk-neutral expected instantaneous return equals the risk-free shadow short rate, $s_{t}$, which may be negative. In contrast, in the world with currency, the price at time $t$ for a zero-coupon bond that pays $\$ 1$ when it matures in $\tau$ years is given by $\underline{P}_{t}(\tau)$. This price will never rise above par, so nonnegative yields will never be observed.

Now consider the relationship between the two bond prices at time $t$ for the shortest (say, overnight) maturity available, $\delta$. In the presence of currency, investors can either buy the zerocoupon bond at price $P_{t}(\delta)$ and receive one unit of currency the following day or just hold the currency. As a consequence, this bond price, which would equal the shadow bond price, must be capped at 1 :

$$
\begin{aligned}
\underline{P}_{t}(\delta) & =\min \left\{1, P_{t}(\delta)\right\} \\
& =P_{t}(\delta)-\max \left\{P_{t}(\delta)-1,0\right\} .
\end{aligned}
$$

That is, the availability of currency implies that the overnight claim has a value equal to the zerocoupon shadow bond price minus the value of a call option on the zero-coupon shadow bond with a strike price of 1 . More generally, we can express the price of a bond in the presence of currency as the price of a shadow bond minus the call option on values of the bond above par:

$$
\underline{P}_{t}(\tau)=P_{t}(\tau)-C_{t}^{A}(\tau, \tau ; 1)
$$

where $C_{t}^{A}(\tau, \tau ; 1)$ is the value of an American call option at time $t$ with maturity in $\tau$ years and strike price 1 written on the shadow bond maturing in $\tau$ years. In essence, in a world with currency, the bond investor has had to sell off the possible gain from the bond rising above par at any time prior to maturity.

\footnotetext{
${ }^{13}$ Both Kim and Singleton (2012) and Bomfim (2003) use finite-difference methods to calculate bond prices, while Ichiue and Ueno (2007) employ interest rate lattices.
} 
Unfortunately, analytically valuing this American option is complicated by the difficulty in determining the early exercise premium. However, Krippner (2013) argues that there is an analytically close approximation based on tractable European options. Specifically, he argues that the above discussion suggests that the last incremental forward rate of any bond will be nonnegative due to the future availability of currency in the immediate time prior to its maturity. As a consequence, he introduces the following auxiliary bond price equation

$$
P_{t}^{\text {aux. }}(\tau, \tau+\delta)=P_{t}(\tau+\delta)-C_{t}^{E}(\tau, \tau+\delta ; 1)
$$

where $C_{t}^{E}(\tau, \tau+\delta ; 1)$ is the value of a European call option at time $t$ with maturity $t+\tau$ and strike price 1 written on the shadow discount bond maturing at $t+\tau+\delta$. It should be stressed that $P_{t}^{\text {aux. }}(\tau, \tau+\delta)$ is not identical to the bond price $\underline{P}_{t}(\tau)$ in equation (8) whose yield observes the zero lower bound.

The key insight of Krippner is that the last incremental forward rate of any bond will be nonnegative due to the future availability of currency in the immediate time prior to its maturity. In Equation (9), this is obtained by letting $\delta \rightarrow 0$, which identifies the corresponding nonnegative instantaneous forward rate:

$$
\underline{f}_{t}(\tau)=\lim _{\delta \rightarrow 0}\left[-\frac{\partial}{\partial \delta} \ln P_{t}^{a u x} \cdot(\tau, \tau+\delta)\right] .
$$

Now, the discount bond prices whose yields observe the zero lower bound are approximated by

$$
\underline{P}_{t}^{a p p \cdot}(\tau)=e^{-\int_{t}^{t+\tau} \underline{f}_{t}(s) d s}
$$

The auxiliary bond price drops out of the calculations, and we are left with formulas for the nonnegative forward rate, $\underline{f}_{t}(\tau)$, that are solely determined by the properties of the shadow rate process $s_{t}$. Specifically, Krippner (2013) shows that

$$
\underline{f}_{t}(\tau)=f_{t}(\tau)+z_{t}(\tau)
$$

where $f_{t}(\tau)$ is the instantaneous forward rate on the shadow bond, which may go negative, while $z_{t}(\tau)$ is given by

$$
z_{t}(\tau)=\lim _{\delta \rightarrow 0}\left[\frac{\partial}{\partial \delta} \frac{C_{t}^{E}(\tau, \tau+\delta ; 1)}{P_{t}(\tau+\delta)}\right] .
$$

In addition, it holds that the observed instantaneous risk-free rate respects the nonnegativity equation (17) as in the Black (1995) model. 
Finally, yield-to-maturity is defined the usual way as

$$
\begin{aligned}
\underline{y}_{t}(\tau) & =\frac{1}{\tau} \int_{t}^{t+\tau} \underline{f}_{t}(s) d s \\
& =\frac{1}{\tau} \int_{t}^{t+\tau} f_{t}(s) d s+\frac{1}{\tau} \int_{t}^{t+\tau} \lim _{\delta \rightarrow 0}\left[\frac{\partial}{\partial \delta} \frac{C_{t}^{E}(s, s+\delta ; 1)}{P_{t}(s+\delta)}\right] d s \\
& =y_{t}(\tau)+\frac{1}{\tau} \int_{t}^{t+\tau} \lim _{\delta \rightarrow 0}\left[\frac{\partial}{\partial \delta} \frac{C_{t}^{E}(s, s+\delta ; 1)}{P_{t}(s+\delta)}\right] d s .
\end{aligned}
$$

It follows that bond yields constrained at the ZLB can be viewed as the sum of the yield on the unconstrained shadow bond, denoted $y_{t}(\tau)$, which is modeled using standard tools, and an add-on correction term derived from the price formula for the option written on the shadow bond that provides an upward push to deliver the higher nonnegative yields actually observed.

It is important to stress that since the observed discount bond prices defined in equation (11) differ from the auxiliary bond price $P_{t}^{a u x} \cdot(\tau, \tau+\delta)$ defined in equation (9) and used in the construction of the nonnegative forward rate in equation (10), the Krippner (2013) framework should be viewed as not fully internally consistent and simply an approximation to an arbitrage-free model 14 Of course, away from the ZLB, with a negligible call option, the model will match the standard arbitrage-free term structure representation. In addition, the size of the approximation error near the ZLB can be determined via simulation as we will demonstrate below.

\subsection{The Shadow-Rate B-CR Model}

In theory, the option-based shadow-rate result is quite general and applies to any assumptions made about the dynamics of the shadow-rate process. However, as implementation requires the calculation of the limit in equation (12), the option-based shadow-rate models are limited practically to the Gaussian model class. The AFNS class is well suited for this extension 15 In the shadow-rate AFNS model, the affine short rate equation (3) is replaced by the nonnegativity constraint and the shadow risk-free rate, which is defined as the sum of level and slope as in the original AFNS model class:

$$
r_{t}=\max \left\{0, s_{t}\right\}, s_{t}=L_{t}+S_{t}
$$

All other elements of the model remain the same. Namely, the dynamics of the state variables used for pricing under the $Q$-measure remain as described in equation (2), so the yield on the shadow

\footnotetext{
${ }^{14}$ In particular, there is no explicit PDE that bond prices must satisfy, including boundary conditions, for the absence of arbitrage as in Kim and Singleton (2012).

${ }^{15}$ For details of the derivations, see Christensen and Rudebusch (2013).
} 
discount bond maintains the popular Nelson and Siegel (1987) factor loading structure

$$
y_{t}(\tau)=L_{t}+\left(\frac{1-e^{-\lambda \tau}}{\lambda \tau}\right) S_{t}+\left(\frac{1-e^{-\lambda \tau}}{\lambda \tau}-e^{-\lambda \tau}\right) C_{t}-\frac{A(\tau)}{\tau}
$$

where $A(\tau) / \tau$ is the same maturity-dependent yield-adjustment term.

The corresponding instantaneous shadow forward rate is given by

$$
f_{t}(\tau)=-\frac{\partial}{\partial T} \ln P_{t}(\tau)=L_{t}+e^{-\lambda \tau} S_{t}+\lambda \tau e^{-\lambda \tau} C_{t}+A^{f}(\tau),
$$

where the yield-adjustment term in the instantaneous forward rate function is given by

$$
\begin{aligned}
A^{f}(\tau)= & -\frac{\partial A(\tau)}{\partial \tau} \\
= & -\frac{1}{2} \sigma_{11}^{2} \tau^{2}-\frac{1}{2}\left(\sigma_{21}^{2}+\sigma_{22}^{2}\right)\left(\frac{1-e^{-\lambda \tau}}{\lambda}\right)^{2} \\
& -\frac{1}{2}\left(\sigma_{31}^{2}+\sigma_{32}^{2}+\sigma_{33}^{2}\right)\left[\frac{1}{\lambda^{2}}-\frac{2}{\lambda^{2}} e^{-\lambda \tau}-\frac{2}{\lambda} \tau e^{-\lambda \tau}+\frac{1}{\lambda^{2}} e^{-2 \lambda \tau}+\frac{2}{\lambda} \tau e^{-2 \lambda \tau}+\tau^{2} e^{-2 \lambda \tau}\right] \\
& -\sigma_{11} \sigma_{21} \tau \frac{1-e^{-\lambda \tau}}{\lambda}-\sigma_{11} \sigma_{31}\left[\frac{1}{\lambda} \tau-\frac{1}{\lambda} \tau e^{-\lambda \tau}-\tau^{2} e^{-\lambda \tau}\right] \\
& -\left(\sigma_{21} \sigma_{31}+\sigma_{22} \sigma_{32}\right)\left[\frac{1}{\lambda^{2}}-\frac{2}{\lambda^{2}} e^{-\lambda \tau}-\frac{1}{\lambda} \tau e^{-\lambda \tau}+\frac{1}{\lambda^{2}} e^{-2 \lambda \tau}+\frac{1}{\lambda} \tau e^{-2 \lambda \tau}\right] .
\end{aligned}
$$

Krippner (2013) provides a formula for the zero lower bound instantaneous forward rate, $\underline{f}_{t}(\tau)$, that applies to any Gaussian model

$$
\underline{f}_{t}(\tau)=f_{t}(\tau) \Phi\left(\frac{f_{t}(\tau)}{\omega(\tau)}\right)+\omega(\tau) \frac{1}{\sqrt{2 \pi}} \exp \left(-\frac{1}{2}\left[\frac{f_{t}(\tau)}{\omega(\tau)}\right]^{2}\right)
$$

where $\Phi(\cdot)$ is the cumulative probability function for the standard normal distribution, $f_{t}(\tau)$ is the shadow forward rate, and $\omega(\tau)$ is related to the conditional variance, $v(\tau, \tau+\delta)$, appearing in the shadow bond option price formula as follows

$$
\omega(\tau)^{2}=\frac{1}{2} \lim _{\delta \rightarrow 0} \frac{\partial^{2} v(\tau, \tau+\delta)}{\partial \delta^{2}}
$$




\begin{tabular}{|c|ccc||c||c|c|}
\hline$K^{P}$ & $K_{\cdot, 1}^{P}$ & $K_{\cdot, 2}^{P}$ & $K_{\cdot, 3}^{P}$ & $\theta^{P}$ & & $\Sigma$ \\
\hline$K_{1, \cdot}^{P}$ & $10^{-7}$ & 0 & 0 & 0 & $\sigma_{11}$ & 0.0067 \\
& & & & & & $(0.0001)$ \\
$K_{2, \cdot}^{P}$ & 0.2892 & 0.3402 & -0.3777 & 0.0214 & $\sigma_{22}$ & 0.0108 \\
& $(0.1533)$ & $(0.1334)$ & $(0.0908)$ & $(0.0343)$ & & $(0.0002)$ \\
$K_{3, \cdot}^{P}$ & 0 & 0 & 0.5153 & -0.0271 & $\sigma_{33}$ & 0.0262 \\
& & & $(0.1252)$ & $(0.0085)$ & & $(0.0004)$ \\
\hline
\end{tabular}

\section{Table 2: Parameter Estimates for the B-CR Model.}

The estimated parameters of the $K^{P}$ matrix, $\theta^{P}$ vector, and diagonal $\Sigma$ matrix are shown for the B-CR model. The estimated value of $\lambda$ is 0.4673 (0.0027). The numbers in parentheses are the estimated parameter standard deviations. The maximum log likelihood value is $66,755.04$.

Within the shadow-rate AFNS model, $\omega(\tau)$ takes the following form

$$
\begin{aligned}
\omega(\tau)^{2}= & \sigma_{11}^{2} \tau+\left(\sigma_{21}^{2}+\sigma_{22}^{2}\right) \frac{1-e^{-2 \lambda \tau}}{2 \lambda} \\
& +\left(\sigma_{31}^{2}+\sigma_{32}^{2}+\sigma_{33}^{2}\right)\left[\frac{1-e^{-2 \lambda \tau}}{4 \lambda}-\frac{1}{2} \tau e^{-2 \lambda \tau}-\frac{1}{2} \lambda \tau^{2} e^{-2 \lambda \tau}\right] \\
& +2 \sigma_{11} \sigma_{21} \frac{1-e^{-\lambda \tau}}{\lambda}+2 \sigma_{11} \sigma_{31}\left[-\tau e^{-\lambda \tau}+\frac{1-e^{-\lambda \tau}}{\lambda}\right] \\
& +\left(\sigma_{21} \sigma_{31}+\sigma_{22} \sigma_{32}\right)\left[-\tau e^{-2 \lambda \tau}+\frac{1-e^{-2 \lambda \tau}}{2 \lambda}\right]
\end{aligned}
$$

Therefore, the zero-coupon bond yields that observe the zero lower bound, denoted $\underline{y}_{t}(\tau)$, are easily calculated as

$$
\underline{y}_{t}(\tau)=\frac{1}{\tau} \int_{t}^{t+\tau}\left[f_{t}(s) \Phi\left(\frac{f_{t}(s)}{\omega(s)}\right)+\omega(s) \frac{1}{\sqrt{2 \pi}} \exp \left(-\frac{1}{2}\left[\frac{f_{t}(s)}{\omega(s)}\right]^{2}\right)\right] d s .
$$

As in the affine AFNS model, the shadow-rate AFNS model is completed by specifying the price of risk using the essentially affine risk premium specification introduced by Duffee (2002), so the realworld dynamics of the state variables can be expressed as equation (5). Again, in an unrestricted case, both $K^{P}$ and $\theta^{P}$ are allowed to vary freely relative to their counterparts under the $Q$-measure. However, we focus on the case with the same $K^{P}$ and $\theta^{P}$ restrictions as in the CR model on the assumption that outside of the ZLB period, the shadow-rate model would properly collapse to the standard CR form. We label this shadow-rate model the "B-CR model."

We estimate the B-CR model from January 4, 1985, until December 28, 2012, for eight maturities: three months, six months, one year, two years, three years, five years, seven years, and ten years 16

\footnotetext{
${ }^{16}$ Due to the nonlinear measurement equation for the yields in the shadow-rate AFNS model, estimation is based on the standard extended Kalman filter as described in Christensen and Rudebusch (2013). We also estimated unrestricted and independent factor shadow-rate AFNS models and obtained similar results to those reported below.
} 
The estimated B-CR model parameters are reported in Table 2, In comparing the estimated B-CR and CR model parameters, we note that none of the parameters are statistically significantly different from the corresponding parameter in the other model with the exception of $\lambda$, which is statistically, but not economically, different across the two models. Hence, for parsimoniously specified Gaussian models, we conjecture that the differences in the estimated parameters between two otherwise identical models that are only distinguished by one being a standard model and the other being a shadow-rate model would tend to be small.

\subsection{How Good is the Option-Based Approximation?}

As noted in Section 3.1, Krippner (2013) does not provide a formal derivation of arbitrage-free pricing relationships for the option-based approach. Therefore, in this subsection, we analyze how closely the option-based bond pricing from the estimated B-CR model matches an arbitrage-free bond pricing that is obtained from the same model using Black's (1995) approach based on Monte Carlo simulations. The simulation-based shadow yield curve is obtained from 50,000 ten-year long factor paths generated using the estimated $Q$-dynamics of the state variables in the B-CR model, which, ignoring the nonnegativity equation (7), are used to construct 50,000 paths for the shadow short rate. These are converted into a corresponding number of shadow discount bond paths and averaged for each maturity before the resulting shadow discount bond prices are converted into yields. The simulation-based yield curve is obtained from the same underlying 50,000 Monte Carlo factor paths, but at each point in time in the simulation, the resulting short rate is constrained by the nonnegativity equation (7) as in Black (1995). The shadow-rate curve from the B-CR model can also be calculated analytically via the usual affine pricing relationships, which ignore the ZLB. Thus, any difference between these two curves is simply numerical error that reflects the finite number of simulations.

To document that the close match between the option-based and the simulation-based yield curves is not limited to any specific date where the ZLB of nominal yields is likely to have mattered, we undertake this simulation exercise for the last observation date in each year since 2007.17 Table 3 reports the resulting shadow yield curve differences and yield curve differences for various maturities on these 7 dates. Note that the errors for the shadow yield curves solely reflect simulation error as the model-implied shadow yield curve is identical to the analytical arbitrage-free curve that would prevail without currency in circulation. These simulation errors in Table 3 are typically very small in absolute value, and they increase only slowly with maturity. Their average absolute value - shown in the bottom row - is less than one basis point even at a ten-year maturity. This implies that using simulations with a large number of draws $(N=50,000)$ arguably delivers enough accuracy for the

\footnotetext{
${ }^{17}$ Of course, away from the ZLB, with a negligible call option, the model will match the standard arbitrage-free term structure representation.
} 


\begin{tabular}{llccccc}
\hline \multirow{2}{*}{ Dates } & & \multicolumn{5}{c}{ Maturity in months } \\
\cline { 3 - 7 } & & 12 & 36 & 60 & 84 & 120 \\
\hline \hline \multirow{2}{*}{$12 / 29 / 06$} & Shadow yields & 0.09 & -0.11 & 0.04 & -0.06 & -0.35 \\
& Yields & 0.12 & -0.11 & 0.08 & 0.15 & 0.17 \\
\hline \multirow{2}{*}{$12 / 28 / 07$} & Shadow yields & 0.30 & 0.31 & 0.09 & 0.06 & 0.23 \\
& Yields & 0.35 & 0.37 & 0.30 & 0.44 & 0.84 \\
\hline \multirow{2}{*}{$12 / 26 / 08$} & Shadow yields & 0.09 & 0.81 & 1.04 & 0.74 & 0.24 \\
& Yields & 0.13 & 0.83 & 1.66 & 1.91 & 2.02 \\
\hline \multirow{2}{*}{$12 / 31 / 09$} & Shadow yields & 0.29 & 0.24 & 0.38 & 0.58 & 0.75 \\
& Yields & 0.25 & 0.43 & 0.81 & 1.18 & 1.50 \\
\hline \multirow{2}{*}{$12 / 31 / 10$} & Shadow yields & -0.31 & -0.37 & 0.26 & 0.95 & 1.44 \\
& Yields & -0.24 & 0.06 & 1.08 & 2.05 & 2.83 \\
\hline \multirow{2}{*}{$12 / 30 / 11$} & Shadow yields & 0.14 & 0.08 & -0.45 & -0.66 & -0.77 \\
& Yields & 0.16 & 0.51 & 0.99 & 1.69 & 2.74 \\
\hline \multirow{2}{*}{$12 / 28 / 12$} & Shadow yields & -0.23 & -0.16 & 0.14 & -0.09 & -0.18 \\
& Yields & -0.03 & 0.27 & 1.35 & 2.13 & 3.25 \\
\hline \hline \multirow{2}{*}{ Average } & Shadow yields & 0.21 & 0.30 & 0.34 & 0.45 & 0.57 \\
abs. diff. & Yields & 0.18 & 0.37 & 0.90 & 1.36 & 1.91 \\
\hline
\end{tabular}

Table 3: Approximation Errors in Yields for Shadow-Rate Model.

At each date, the table reports differences between the analytical shadow yield curve obtained from the option-based estimates of the B-CR model and the shadow yield curve obtained from 50,000 simulations of the estimated factor dynamics under the $Q$-measure in that model. The table also reports for each date the corresponding differences between the fitted yield curve obtained from the B-CR model and the yield curve obtained via simulation of the estimated B-CR model with imposition of the ZLB. The bottom two rows give averages of the absolute differences across the 7 dates. All numbers are measured in basis points.

type of inference we want to make here.

Given this calibration of the size of the numerical errors involved in the simulation, we can now assess the more interesting size of the approximation error in the option-based approach to valuing yields in the presence of the ZLB. In Table 3, the errors of the fitted B-CR model yield curves relative to the simulated results are only slightly larger than those reported for the shadow yield curve. In particular, for maturities up to five years, the errors tend to be less than 1 basis point, so the optionbased approximation error adds very little if anything to the numerical simulation error. At the ten-year maturity, the approximation errors are understandably larger, but even the largest errors at the ten-year maturity do not exceed 4 basis points in absolute value and the average absolute value is less than 2 basis points. Overall, the option-based approximation errors in our three-factor setting appear relatively small. Indeed, they are smaller than the fitted errors to be reported in Table 4 , That is, for the B-CR model analyzed here, the gain from using a numerical estimation approach instead of the option-based approximation would in all likelihood be negligible. 


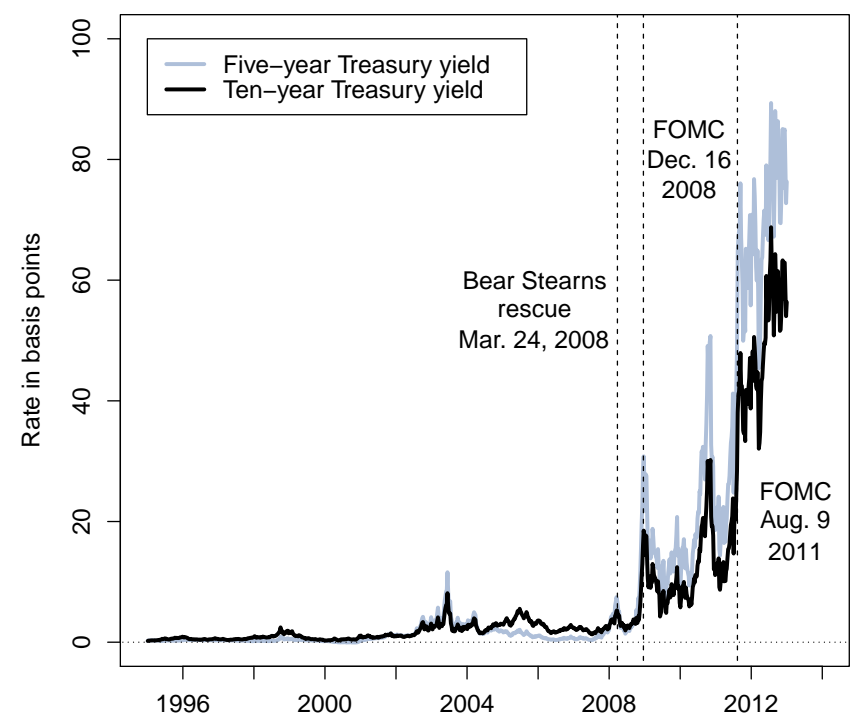

Figure 3: Value of Option to Hold Currency.

We show time-series plots of the value of the option to hold currency embedded in the Treasury yield curve as estimated in real time by the B-CR model. The data cover the period from January 6, 1995, to December $28,2012$.

\subsection{Measuring the Effect of the ZLB}

To provide evidence that we should anticipate to see at least some difference across the regular and shadow-rate models, we turn our focus to the value of the option to hold currency, which we define as the difference between the yields that observe the zero lower bound and the comparable lower shadow discount bond yields that do not. Figure 3 shows these yield spreads at the fiveand ten-year maturity based on real-time rolling weekly re-estimations of the B-CR model starting in 1995 through 2012. Beyond a few very temporary small spikes, the option was of economically insignificant value prior to the failure of Lehman Brothers in the fall of 2008 18 However, despite the zero short rate since 2008, it is not really until August 2011 that the option obtains significant sustained value. At the end of our sample, the yield spread is 80 and 60 basis points at the five- and ten-year maturity, respectively. Option values at those levels suggest that it should matter for model performance whether a model accounts for the ZLB of nominal yields.

\footnotetext{
${ }^{18}$ Consistent with our series for the 2003-period, Bomfim (2003) in his calibration of a two-factor shadow-rate model to U.S. interest rate swap data reports a probability of hitting the zero-boundary within the next two years equaling 3.6 percent as of January 2003. Thus, it appears that there was never any material risk of reaching the ZLB during the 2003-2004 period of low interest rates.
} 


\begin{tabular}{|ccccccccccc|}
\hline \multicolumn{7}{|c|}{ Mull sample } & All \\
\cline { 1 - 9 } RMSE & 3 & 6 & 12 & 24 & 36 & 60 & 84 & 120 & yields \\
\cline { 2 - 9 } & 31.45 & 15.23 & 0.0 & 2.41 & 0.00 & 3.02 & 2.74 & 10.59 & 13.02 \\
CR & 30.89 & 14.74 & 0.7 & 2.27 & 0.25 & 2.74 & 2.43 & 10.10 & 12.71 \\
\hline
\end{tabular}

\begin{tabular}{|c|c|c|c|c|c|c|c|c|c|}
\hline \multicolumn{10}{|c|}{ Normal period (Jan. 6, 1995-Dec. 12, 2008) } \\
\hline \multirow{2}{*}{ RMSE } & \multicolumn{8}{|c|}{ Maturity in months } & \multirow{2}{*}{$\begin{array}{c}\text { All } \\
\text { yields }\end{array}$} \\
\hline & 3 & 6 & 12 & 24 & 36 & 60 & 84 & 120 & \\
\hline $\mathrm{CR}$ & 32.73 & 15.65 & 0.00 & 2.51 & 0.00 & 3.00 & 2.56 & 10.56 & 13.46 \\
\hline $\mathrm{B}-\mathrm{CR}$ & 32.74 & 15.53 & 0.59 & 2.40 & 0.09 & 2.83 & 2.23 & 10.49 & 13.43 \\
\hline
\end{tabular}

\begin{tabular}{|c|c|c|c|c|c|c|c|c|c|}
\hline \multicolumn{10}{|c|}{ ZLB period (Dec. 19, 2008-Dec. 28, 2012) } \\
\hline \multirow{2}{*}{ RMSE } & \multicolumn{8}{|c|}{ Maturity in months } & \multirow{2}{*}{$\begin{array}{c}\text { All } \\
\text { yields }\end{array}$} \\
\hline & 3 & 6 & 12 & 24 & 36 & 60 & 84 & 120 & \\
\hline CR & 22.40 & 12.53 & 0.00 & 1.73 & 0.00 & 3.11 & 3.66 & 10.78 & 10.01 \\
\hline $\mathrm{B}-\mathrm{CR}$ & 16.03 & 8.75 & 1.15 & 1.23 & 0.61 & 2.13 & 3.37 & 7.37 & 7.13 \\
\hline
\end{tabular}

Table 4: Summary Statistics of the Fitted Errors.

The root mean squared fitted errors (RMSEs) for the CR and B-CR model are shown. All numbers are measured in basis points. The data covers the period from January 6, 1985, to December 28, 2012.

\section{Comparing Affine and Shadow-Rate Models}

In this section, we compare the empirical affine and shadow-rate models across a variety of dimensions, including in-sample fit, volatility dynamics, and out-of-sample forecast performance.

\subsection{In-Sample Fit and Volatility}

The summary statistics of the model fit are reported in Table 4 and indicate a very similar fit of the two models in the normal period up until the end of 2008. However, since then we see a notable advantage to the shadow-rate model that is also reflected in the likelihood values. Still, we conclude from this in-sample analysis that it is not in the model parameters nor in the model fit that the shadow-rate model really distinguishes itself from its regular cousin.

A serious limitation of standard Gaussian models is the assumption of constant yield volatility, which is particularly unrealistic when periods of normal volatility are combined with periods in which yields are greatly constrained in their movements near the ZLB. A shadow-rate model approach can mitigate this failing significantly.

In the CR model, where zero-coupon yields are affine functions of the state variables, model- 


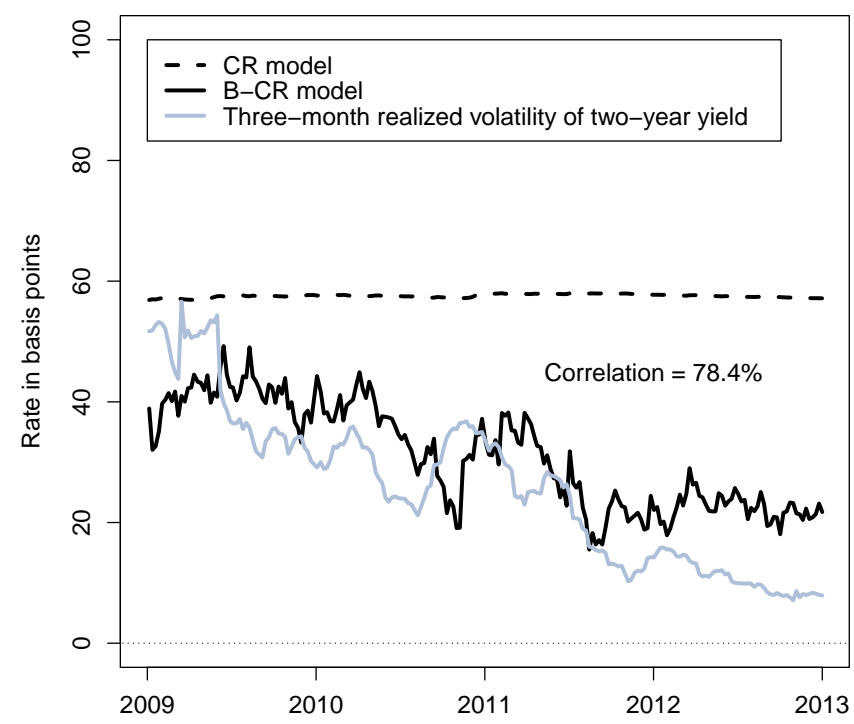

Figure 4: Three-Month Conditional Volatility of Two-Year Yield Since 2009.

Illustration of the three-month conditional volatility of the two-year yield implied by the estimated CR and B-CR model. Also shown is the subsequent three-month realized volatility of the two-year yield based on daily data.

implied conditional predicted yield volatilities are given by the square root of

$$
V_{t}^{P}\left[y_{T}^{N}(\tau)\right]=\frac{1}{\tau^{2}} B(\tau)^{\prime} V_{t}^{P}\left[X_{T}\right] B(\tau)
$$

where $T-t$ is the prediction period, $\tau$ is the yield maturity, and $V_{t}^{P}\left[X_{T}\right]$ is the conditional covariance matrix 19 In the B-CR model, on the other hand, zero-coupon yields are non-linear functions of the state variables and conditional predicted yield volatilities have to be generated by standard Monte Carlo simulation. Figure 4 shows the implied three-month conditional yield volatility of the two-year yield from the CR and B-CR models.

To evaluate the fit of these predicted three-month-ahead conditional yield standard deviations, they are compared to a standard measure of realized volatility based on the same data used in the model estimation, but at daily frequency. The realized standard deviation of the daily changes in the interest rates are generated for the 91-day period ahead on a rolling basis. The realized variance measure is used by Andersen and Benzoni (2010), Collin-Dufresne et al. (2009), as well as Jacobs and Karoui (2009) in their assessments of stochastic volatility models. This measure is

\footnotetext{
${ }^{19}$ The conditional covariance matrix is calculated using the analytical solutions provided in Fisher and Gilles (1996).
} 
fully nonparametric and has been shown to converge to the underlying realization of the conditional variance as the sampling frequency increases; see Andersen et al. (2003) for details. The square root of this measure retains these properties. For each observation date $t$ the number of trading days $N$ during the subsequent 91-day time window is determined and the realized standard deviation is calculated as

$$
R V_{t, \tau}^{S T D}=\sqrt{\sum_{n=1}^{N} \Delta y_{t+n}^{2}(\tau)},
$$

where $\Delta y_{t+n}(\tau)$ is the change in yield $y(\tau)$ from trading day $t+(n-1)$ to trading day $t+n 20$

While the conditional yield volatility from the CR model only change little (due to updating of its estimated parameters), the conditional yield volatility from the B-CR model closely matches the realized volatility series 21

\subsection{Forecast Performance}

To extract the term premiums embedded in the Treasury yield curve is ultimately an exercise in forecasting policy expectations. Thus, to study bond investors' expectations in real time, we perform a rolling re-estimation of the $\mathrm{CR}$ model and its shadow-rate equivalent on expanding samples - adding one week of observations each time, a total of 939 estimations. As a result, the end dates of the expanding samples run from January 6, 1995, to December 28, 2012. For each end date during that period, we project the short rate six months and one year ahead. Importantly, the estimates of these objects rely essentially only on information that was available in real time.

For robustness, we include results from another established U.S. Treasury term structure model introduced in Kim and Wright (2005, henceforth KW). It is a standard latent three-factor Gaussian term structure model of the kind described in Section $2.1{ }^{22}$ This model is updated on an ongoing basis by the staff of the Federal Reserve Board 23 However, we emphasize that the forecasts from this model are not real-time forecasts, but based on the full sample estimate.

As yields were both economically and statistically far away from the zero lower bound prior to December 2008, it seems reasonable to distinguish between model performance in the normal period prior to the policy rate reaching its effective lower bound and the period after it. For the

\footnotetext{
${ }^{20}$ Note that other measures of realized volatility have been used in the literature, such as the realized mean absolute deviation measure as well as fitted GARCH estimates. Collin-Dufresne et al. (2009) also use option-implied volatility as a measure of realized volatility.

${ }^{21}$ In their analysis of Japanese government bond yields, Kim and Singleton (2012) also report a close match to yield volatilities for their Gaussian shadow-rate model.

${ }^{22}$ The KW model is estimated using one-, two-, four-, seven-, and ten-year off-the-run Treasury zero-coupon yields from the Gürkaynak et al. (2007) database, as well as three- and six-month Treasury bill yields. To facilitate empirical implementation, model estimation includes monthly data on the six- and twelve-month-ahead forecasts of the threemonth T-bill yield from Blue Chip Financial Forecasts and semiannual data on the average expected three-month T-bill yield six to eleven years hence from the same source. See Kim and Orphanides (2012) for details.

${ }^{23}$ The data is available at www.federalreserve.gov.
} 


\begin{tabular}{|l|c|c||c|c|}
\hline \multirow{2}{*}{ Full forecast period } & \multicolumn{2}{|c||}{ Six-month forecast } & \multicolumn{2}{c|}{ One-year forecast } \\
\cline { 2 - 5 } & Mean & RMSE & Mean & RMSE \\
\hline Random walk & 16.51 & 84.11 & 33.43 & 150.27 \\
KW model & 4.09 & 64.32 & 45.90 & 126.56 \\
CR model & -2.14 & 65.62 & 10.23 & 128.64 \\
B-CR model & 3.28 & 62.70 & 22.23 & 123.98 \\
\hline
\end{tabular}

\begin{tabular}{|l|c|c||c|c|}
\hline \multirow{2}{*}{ Normal forecast period } & \multicolumn{2}{|c||}{ Six-month forecast } & \multicolumn{2}{c|}{ One-year forecast } \\
\cline { 2 - 5 } & Mean & RMSE & Mean & RMSE \\
\hline Random walk & 20.71 & 94.19 & 40.73 & 165.87 \\
KW model & -3.40 & 69.68 & 35.62 & 132.67 \\
CR model & -0.33 & 72.08 & 16.41 & 141.20 \\
B-CR model & 2.57 & 69.97 & 24.00 & 136.56 \\
\hline
\end{tabular}

\begin{tabular}{|l|c|c||c|c|}
\hline \multirow{2}{*}{ ZLB forecast period } & \multicolumn{2}{|c||}{ Six-month forecast } & \multicolumn{2}{c|}{ One-year forecast } \\
\cline { 2 - 5 } & Mean & RMSE & Mean & RMSE \\
\hline Random walk & 0.00 & 0.00 & 0.00 & 0.00 \\
KW model & 33.55 & 36.21 & 92.94 & 93.59 \\
CR model & -9.26 & 28.37 & -18.10 & 32.24 \\
B-CR model & 6.06 & 11.52 & 14.09 & 19.10 \\
\hline
\end{tabular}

Table 5: Summary Statistics for Target Federal Funds Rate Forecast Errors.

Summary statistics of the forecast errors - mean and root mean-squared errors (RMSEs) - of the target overnight federal funds rate six months and one year ahead. The forecasts are weekly. The top panel covers the full forecast period that starts on January 6, 1995, and runs until June 29, 2012, for the six-month forecasts (913 forecasts) and until December 30, 2011, for the one-year forecasts (887 forecasts). The middle panel coves the normal forecast period from January 6, 1995, to December 12, 2008, 728 forecasts. The lower panel covers the zero lower bound forecast period that starts on December 19, 2008, and runs until June 29, 2012, for the six-month forecasts (185 forecasts) and until December 30, 2011, for the one-year forecasts (159 forecasts). All measurements are expressed in basis points.

13 years from 1995 through 2008 we should anticipate to be able to document essentially identical performance along most dimensions, while we could hope to establish some superior performance for the shadow-rate model in the years since 2009 in terms of forecasting future policy rates up to two years ahead.

The summary statistics for the forecast errors relative to the subsequent realizations of the target overnight federal funds rate set by the FOMC are reported in Table 5. which also contains the forecast errors obtained using a random walk assumption. We note the strong forecast performance of the $\mathrm{KW}$ model relative to the CR model during the normal period 24 while it is equally obvious that the KW model underperforms grossly during the ZLB period since December 19, 2008. As expected, the

\footnotetext{
${ }^{24}$ As noted earlier, this is not an entirely fair race as the KW model is not estimated on a rolling real-time basis unlike the other models.
} 


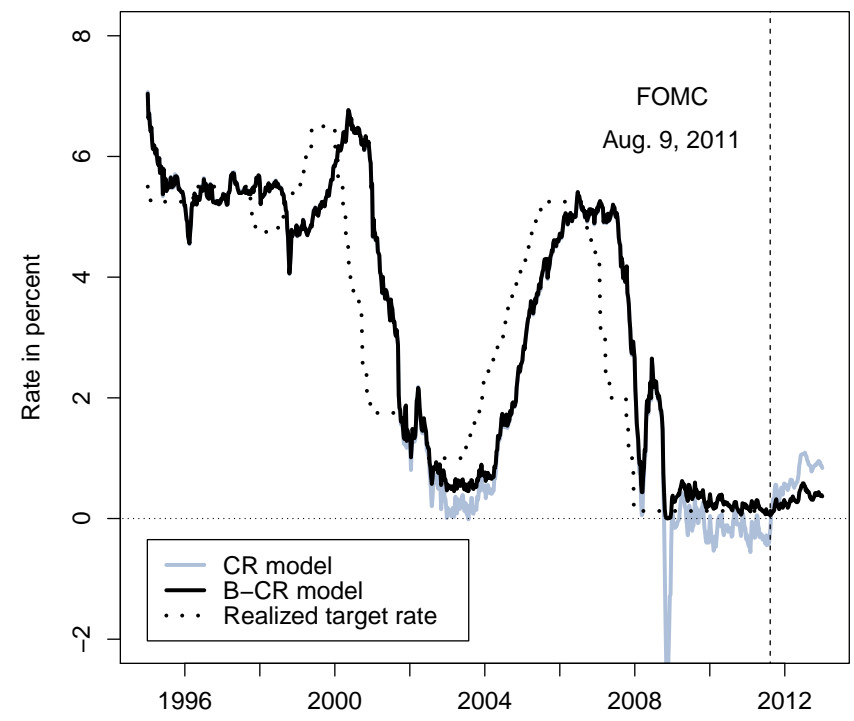

Figure 5: Forecasts of the Target Overnight Federal Funds Rate.

Forecasts of the target overnight federal funds rate one year ahead from the CR and B-CR model. Subsequent realizations of the target overnight federal funds rate are included, so at date $t$, the figure shows forecasts as of time $t$ and the realization from $t$ plus one year. The forecast data are weekly observations from January 6 , 1995, to December 28, 2012.

$\mathrm{CR}$ and B-CR models exhibit very similar performance during the normal period, while the B-CR model stands out in the most recent ZLB period 25

Figure 5 compares the forecasts at the one-year horizon from the CR and B-CR models to the subsequent target rate realizations. For the $\mathrm{CR}$ model, the deterioration in forecast performance is not really detectable until after the August 2011 FOMC meeting when explicit forward guidance was first introduced. Since the CR model mitigates finite-sample bias in the estimates of the meanreversion matrix $K^{P}$ by imposing a unit-root property on the Nelson-Siegel level factor, it suggests that the recent deterioration for the CR model must be caused by other more fundamental factors.

\subsection{Decomposing Ten-Year Yields}

One important use for affine DTSMs has been to separate longer-term yields into a short-rate expectations component and a term premium. Here, we document the different decompositions of

\footnotetext{
${ }^{25}$ As another robustness check, we used the estimated parameters of the CR model combined with filtering from the B-CR model to generate an alternative set of short rate forecasts. The results are very close to those shown in Table 5 for the B-CR model and hence not reported.
} 


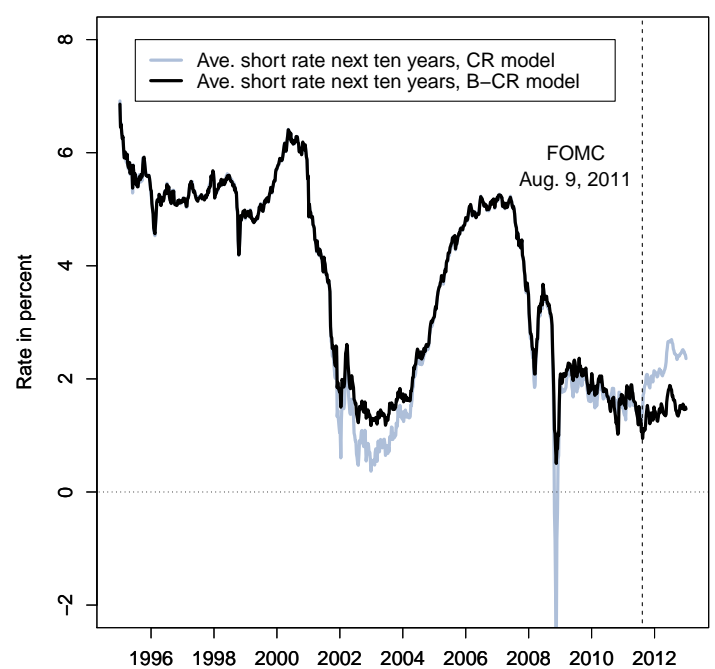

(a) Expected short rate.

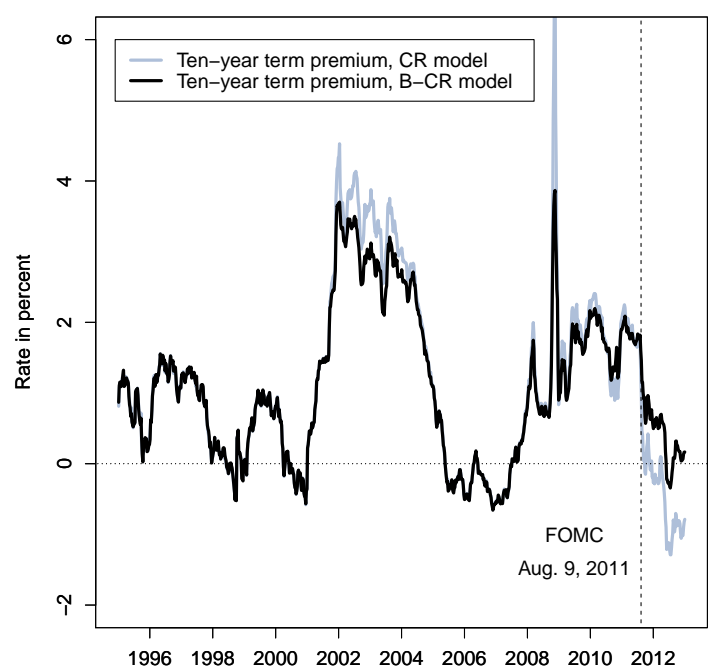

(b) Term premium.

Figure 6: Ten-Year Expected Short Rate and Term Premium

Panel (a) provides real-time estimates of the average policy rate expected over the next ten years from the $\mathrm{CR}$ and B-CR model. Panel (b) shows the corresponding ten-year term premium series. The data cover the period from January 6, 1995, to December 28, 2012.

the ten-year Treasury yield implied by the CR and B-CR model. To do so, we calculate, for each end date during our rolling re-estimation period, the average expected path for the overnight rate, $(1 / \tau) \int_{t}^{t+\tau} E_{t}^{P}\left[r_{s}\right] d s$, as well as the associated term premium - assuming the two components sum to the fitted bond yield, $\widehat{y}_{t}(\tau) 26$

Figure 6 shows the real-time decomposition of the ten-year Treasury yield into a policy expectations component and a term premium component according to the CR and B-CR models. Studying the time-series patterns in greater detail, we first note the similar decompositions from the CR and B-CR model until December 2008 - with the notable exception of the 2002-2004 period when yields were low the last time. Second, we see some smaller discrepancies across these two model decompositions in the period between December 2008 and August 2011. Finally, we point out the sustained difference in the extracted policy expectations and term premiums in the period since August 2011.

These results suggest that at least through late 2011, the ZLB did not greatly effect the term premium decomposition. To provide a concrete example of this, we repeat the analysis in CR of the Treasury yield response to eight key announcements by the Fed regarding its first large-scale asset purchase (LSAP) program. Table 6 shows the CR and B-CR model decompositions of the 10-year U.S. Treasury yield on these eight dates and the total changes. The yield decompositions on these dates are quite similar for both of these models, though the B-CR model ascribes a bit more of the

\footnotetext{
${ }^{26}$ The details of these calculations for both the $\mathrm{CR}$ and B-CR model are provided in Appendices A and B.
} 


\begin{tabular}{|c|c|c|c|c|c|}
\hline \multirow[b]{2}{*}{$\begin{array}{c}\text { Announcement } \\
\text { date }\end{array}$} & \multirow[b]{2}{*}{ Model } & \multicolumn{3}{|c|}{ Decomposition from models } & \multirow{2}{*}{$\begin{array}{c}\text { Ten-year } \\
\text { Treasury } \\
\text { yield }\end{array}$} \\
\hline & & $\begin{array}{c}\text { Avg. target rate } \\
\text { next ten years }\end{array}$ & $\begin{array}{c}\text { Ten-year } \\
\text { term premium }\end{array}$ & Residual & \\
\hline \multirow{2}{*}{ Nov. 25, 2008} & $\overline{\overline{C R}}$ & -20 & 0 & -2 & \multirow{2}{*}{-21} \\
\hline & $\mathrm{B}-\mathrm{CR}$ & -10 & -10 & 0 & \\
\hline \multirow{2}{*}{ Dec. 1, 2008} & $\mathrm{CR}$ & -10 & -10 & -2 & \multirow{2}{*}{-22} \\
\hline & $\mathrm{B}-\mathrm{CR}$ & -21 & 2 & -3 & \\
\hline \multirow{2}{*}{ Dec. 16,2008} & $\overline{\mathrm{CR}}$ & -7 & -7 & -3 & \multirow{2}{*}{-17} \\
\hline & $\mathrm{B}-\mathrm{CR}$ & -17 & 3 & -3 & \\
\hline \multirow{2}{*}{ Jan. 28, 2009} & $\mathrm{CR}$ & 6 & 1 & 5 & \multirow{2}{*}{12} \\
\hline & $\mathrm{B}-\mathrm{CR}$ & 9 & -2 & 5 & \\
\hline \multirow{2}{*}{ Mar. 18, 2009} & $\mathrm{CR}$ & -14 & -23 & -15 & \multirow{2}{*}{-52} \\
\hline & $\mathrm{B}-\mathrm{CR}$ & -17 & -20 & -14 & \\
\hline \multirow{2}{*}{ Aug. 12, 2009} & $\mathrm{CR}$ & -1 & 1 & 6 & \multirow{2}{*}{6} \\
\hline & $\mathrm{B}-\mathrm{CR}$ & -4 & 4 & 6 & \\
\hline \multirow{2}{*}{ Sep. 23, 2009} & $\mathrm{CR}$ & -5 & 2 & 1 & \multirow{2}{*}{-2} \\
\hline & B-CR & -3 & 1 & 1 & \\
\hline \multirow{2}{*}{ Nov. 4, 2009} & $\mathrm{CR}$ & -1 & 5 & 3 & \multirow{2}{*}{7} \\
\hline & $\mathrm{B}-\mathrm{CR}$ & -1 & 5 & 3 & \\
\hline \multirow{2}{*}{ Total net change } & $\overline{\mathrm{CR}}$ & -53 & -29 & -7 & \multirow{2}{*}{-89} \\
\hline & $\mathrm{B}-\mathrm{CR}$ & -65 & -17 & -7 & \\
\hline
\end{tabular}

Table 6: Decomposition of Responses of Ten-Year U.S. Treasury Yield.

The decomposition of responses of the ten-year U.S. Treasury yield on eight LSAP announcement dates into changes in (i) the average expected target rate over the next ten years, (ii) the ten-year term premium, and (iii) the unexplained residual based on the CR and B-CR model of U.S. Treasury yields. All changes are measured in basis points.

changes in yields to a signaling channel effect adjusting short-rate expectations.

\subsection{Assessing Recent Shifts in Near-Term Monetary Policy Expectations}

In this section, we attempt to assess the extent to which the models are able to capture recent shifts in near-term monetary policy expectations.

To do so, we compare the variation in the models' one- and two-year short rate forecasts since 2007 to the rates on one- and two-year federal funds futures contracts as shown in Figure 727 We note that the existence of time-varying risk premiums even in very short-term federal funds futures contracts is well documented (see Piazzesi and Swanson 2008). However, the risk premiums in such short-term contracts are small relative to the sizeable variation over time observed in Figure 7 , As

\footnotetext{
${ }^{27}$ The futures data are from Bloomberg. The one-year futures rate is the weighted average of the rates on the $12-$ and 13-month federal funds futures contracts, while the two-year futures rate is the rate on the 24-month federal funds futures contract through 2010, and the weighted average of the rates on the 24- and 25-month contracts since then. Absence of data on the 24-month contracts prior to 2007 determines the start date for the analysis.
} 


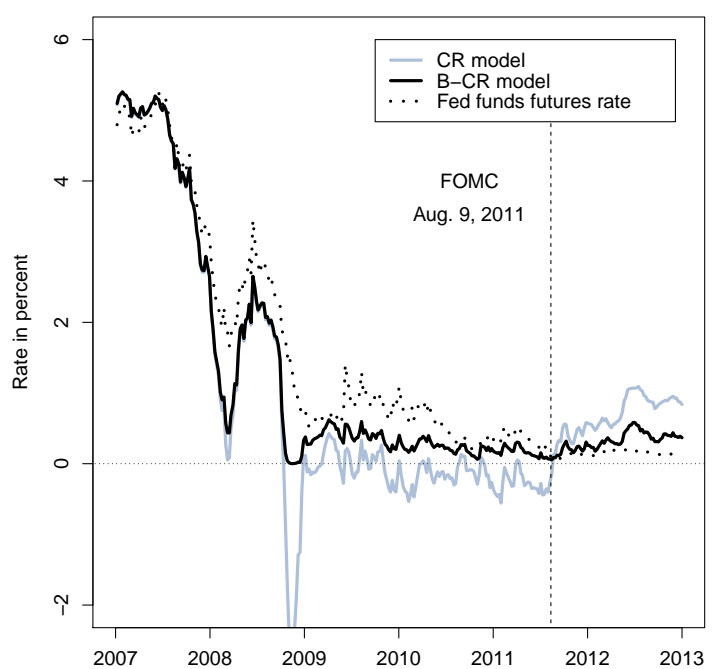

(a) One-year projections.

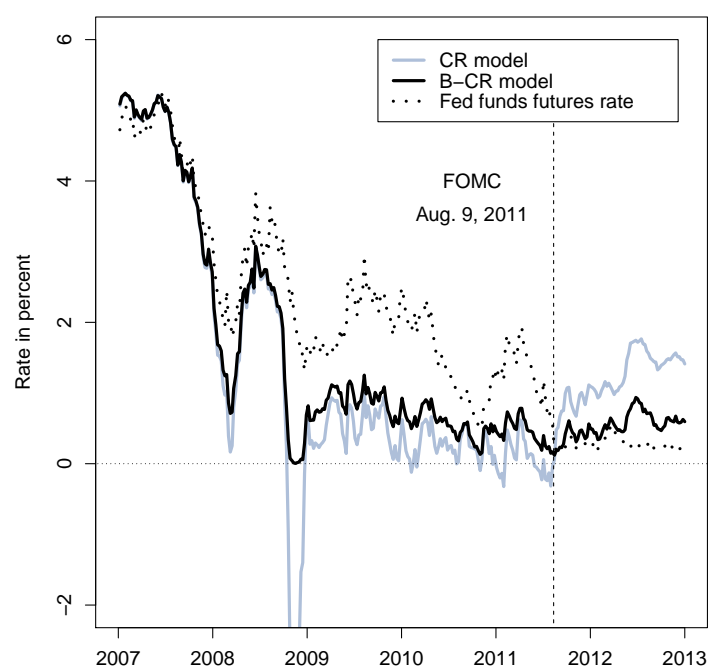

(b) Two-year projections.

Figure 7: Comparison of Short Rate Projections.

Panel (a) illustrates the one-year short rate projections from the CR and B-CR model with a comparison to the rates on one-year federal funds futures. Panel (b) shows the corresponding results for a two-year projection period with a comparison to the rates on two-year federal funds futures. The data are weekly covering the period from January 5, 2007, to December 28, 2012.

a consequence, we interpret the bulk of the variation from 2007 to 2009 as reflecting declines in short rate expectations. Furthermore, since August 2011, most evidence -including our own shown in Figure 6 - suggests that risk premiums have been significantly depressed, likely to a point that a zero-risk-premium assumption for the futures contracts discussed here is a satisfactory approximation. Combined these observations suggest that it is defensible for most of the shown six-year period to map the models' short rate projections to the rates on the federal funds futures contracts without adjusting for their risk premiums.

At the one- and two-year forecast horizons, the correlations between the CR model short rate forecasts and the federal funds futures rates are 0.89 and 0.70 , respectively. The corresponding correlations for the B-CR model are 0.97 and 0.90, respectively. For the CR model, the distance to the futures rates measured by the root mean squared errors (RMSE) are 89.18 and 139.93 basis points at the one- and two-year horizon, respectively, while for the B-CR model the corresponding RMSEs are 46.53 and 84.77 basis points, respectively. Thus, both measured by correlations and by a distance metric, the B-CR model short rate projections appears to be better aligned with the information reflected in rates on federal funds futures than the projections generated by the standard CR model, and this superior performance is particularly clear since August 2011. 


\section{Conclusion}

In this paper, we study the performance of a standard Gaussian DTSM of U.S. Treasury yields and its equivalent shadow-rate version. This provides us with a clean read on the merits of casting a standard model as a shadow-rate model to respect the ZLB of nominal yields.

We find that the standard model performed well until the end of 2008 , while it showed some signs of performance deterioration until August 2011. For the period since then we document notable underperformance of the standard model.

In the current near-ZLB yield environment, we find that the shadow-rate model provides superior in-sample fit, matches the compression in yield volatility unlike the standard model, and delivers better real-time short rate forecasts. Thus, while one could expect the regular model to get back on track as soon as short- and medium-term yields rise from their current low levels, our findings suggest that, in the meantime, shadow-rate models offer a tractable way of mitigating the problems related to the ZLB constraint on nominal yields. Since shadow-rate models collapse to the regular model away from the ZLB, we recommend their use not only when yields are low, but in general.

Finally, we stress that the difference between yield curve dynamics in the normal period and the most recent period with yields near the ZLB could reflect deeper nonlinearities in the factor structure, or maybe even a regime-switch in the model dynamics, that are beyond the static affine dynamic structure assumed in this paper. These remain open questions and we leave them for future research. 


\section{Appendix A: Formula for Policy Expectations in AFNS and B-AFNS Models}

In this appendix, we detail how conditional expectations for future policy rates are calculated within AFNS and B-AFNS models.

In affine models, in general, the conditional expected value of the state variables is calculated as

$$
E_{t}^{P}\left[X_{t+\tau}\right]=\left(I-\exp \left(-K^{P} \tau\right)\right) \theta^{P}+\exp \left(-K^{P} \tau\right) X_{t}
$$

In AFNS models, the instantaneous short rate is defined as

$$
r_{t}=L_{t}+S_{t}
$$

Thus, the conditional expectation of the short rate is

$$
E_{t}^{P}\left[r_{t+\tau}\right]=E_{t}^{P}\left[L_{t+\tau}+S_{t+\tau}\right]=\left(\begin{array}{ccc}
1 & 1 & 0
\end{array}\right) E_{t}^{P}\left[X_{t+\tau}\right] .
$$

In B-AFNS models, the instantaneous shadow rate is defined as

$$
s_{t}=L_{t}+S_{t}
$$

In turn, the conditional expectation of the shadow-rate process is

$$
E_{t}^{P}\left[s_{t+\tau}\right]=E_{t}^{P}\left[L_{t+\tau}+S_{t+\tau}\right]=\left(\begin{array}{ccc}
1 & 1 & 0
\end{array}\right) E_{t}^{P}\left[X_{t+\tau}\right] .
$$

Now, the conditional covariance matrix of the state variables is given by 28

$$
V_{t}^{P}\left[X_{t+\tau}\right]=\int_{0}^{\tau} e^{-K^{P} s} \Sigma \Sigma^{\prime} e^{-\left(K^{P}\right)^{\prime} s} d s
$$

Hence, the conditional covariance of the shadow-rate process is

$$
V_{t}^{P}\left[s_{t+\tau}\right]=\left(\begin{array}{ccc}
1 & 1 & 0
\end{array}\right) V_{t}^{P}\left[X_{t+\tau}\right]\left(\begin{array}{l}
1 \\
1 \\
0
\end{array}\right)
$$

Thus, following equation (65) in Kim and Singleton (2012), the conditional expectation of the short rate in the B-AFNS models,

$$
r_{t}=\max \left(0, s_{t}\right)
$$

is given by

$$
\begin{aligned}
E_{t}^{P}\left[r_{t+\tau}\right] & =\int_{-\infty}^{\infty} f\left(r_{t+\tau} \mid X_{t}\right) d r_{t+\tau}=\int_{0}^{\infty} f\left(s_{t+\tau} \mid X_{t}\right) d s_{t+\tau} \\
& =E_{t}^{P}\left[s_{t+\tau}\right] N\left(\frac{E_{t}^{P}\left[s_{t+\tau}\right]}{\sqrt{V_{t}^{P}\left[s_{t+\tau}\right]}}\right)+\frac{1}{\sqrt{2 \pi}} \sqrt{V_{t}^{P}\left[s_{t+\tau}\right]} \exp \left(-\frac{E_{t}^{P}\left[s_{t+\tau}\right]^{2}}{V_{t}^{P}\left[s_{t+\tau}\right]}\right) .
\end{aligned}
$$

\footnotetext{
${ }^{28}$ We calculate the conditional covariance matrix using the analytical solutions provided in Fisher and Gilles (1996).
} 


\section{Appendix B: Analytical Formulas for Policy Expectations and Term Premiums in the CR Model}

In this appendix, we derive the analytical formulas for policy expectations and term premiums within the CR model.

For a start, the term premium is defined as

$$
T P_{t}(\tau)=y_{t}(\tau)-\frac{1}{\tau} \int_{t}^{t+\tau} E_{t}^{P}\left[r_{s}\right] d s
$$

In the CR model, as in any AFNS model, the instantaneous short rate is defined as

$$
r_{t}=L_{t}+S_{t},
$$

while the specification of the $P$-dynamics is given by

$$
\left(\begin{array}{c}
d L_{t} \\
d S_{t} \\
d C_{t}
\end{array}\right)=\left(\begin{array}{ccc}
10^{-7} & 0 & 0 \\
\kappa_{21}^{P} & \kappa_{22}^{P} & \kappa_{23}^{P} \\
0 & 0 & \kappa_{33}^{P}
\end{array}\right)\left[\left(\begin{array}{c}
0 \\
\theta_{2}^{P} \\
\theta_{3}^{P}
\end{array}\right)-\left(\begin{array}{c}
L_{t} \\
S_{t} \\
C_{t}
\end{array}\right)\right] d t+\left(\begin{array}{ccc}
\sigma_{11} & 0 & 0 \\
0 & \sigma_{22} & 0 \\
0 & 0 & \sigma_{33}
\end{array}\right)\left(\begin{array}{c}
d W_{t}^{L, P} \\
d W_{t}^{S, P} \\
d W_{t}^{C, P}
\end{array}\right) .
$$

Thus, the mean-reversion matrix is given by

$$
K^{P}=\left(\begin{array}{ccc}
10^{-7} & 0 & 0 \\
\kappa_{21}^{P} & \kappa_{22}^{P} & \kappa_{23}^{P} \\
0 & 0 & \kappa_{33}^{P}
\end{array}\right)
$$

Its matrix exponential can be calculated analytically:

$$
\exp \left(-K^{P} \tau\right)=\left(\begin{array}{ccc}
1 & 0 & 0 \\
-\kappa_{21}^{P} \frac{1-e^{-\kappa_{22}^{P} \tau}}{\kappa_{22}^{P}} & e^{-\kappa_{22}^{P} \tau} & -\kappa_{23}^{P} \frac{e^{-\kappa_{33}^{P} \tau}-e^{-\kappa_{22}^{P} \tau}}{\kappa_{22}^{P}-\kappa_{33}^{P}} \\
0 & 0 & e^{-\kappa_{33}^{P} \tau}
\end{array}\right)
$$

Now, the conditional mean of the state variables is

$$
\begin{aligned}
E_{t}^{P}\left[X_{t+\tau}\right] & =\theta^{P}+\left(\begin{array}{ccc}
1 & 0 & 0 \\
-\kappa_{21}^{P} \frac{1-e^{-\kappa_{22}^{P} \tau}}{\kappa_{22}^{P}} & e^{-\kappa_{22}^{P} \tau} & -\kappa_{23}^{P} \frac{e^{-\kappa_{33}^{P} \tau}-e^{-\kappa_{22}^{P} \tau}}{\kappa_{22}^{P}-\kappa_{33}^{P}} \\
0 & 0 & e^{-\kappa_{33}^{P} \tau}
\end{array}\right)\left(\begin{array}{c}
L_{t} \\
S_{t}-\theta_{2}^{P} \\
C_{t}-\theta_{3}^{P}
\end{array}\right) \\
& =\left(\begin{array}{c}
L_{t} \\
\theta_{2}^{P}-\kappa_{21}^{P} \frac{1-e^{-\kappa_{22}^{P} \tau}}{\kappa_{22}^{P}} L_{t}+e^{-\kappa_{22}^{P} \tau}\left(S_{t}-\theta_{2}^{P}\right)-\kappa_{23}^{P} \frac{e^{-\kappa_{33}^{P} \tau}-e^{-\kappa_{22}^{P} \tau}}{\kappa_{22}^{P}-\kappa_{33}^{P}}\left(C_{t}-\theta_{3}^{P}\right) \\
\theta_{3}^{P}+e^{-\kappa_{33}^{P} \tau}\left(C_{t}-\theta_{3}^{P}\right)
\end{array}\right) .
\end{aligned}
$$

In order to get back to the term premium formula, we note that the conditional expectation of the instantaneous short rate process is given by

$$
\begin{aligned}
E_{t}^{P}\left[r_{s}\right] & =E_{t}^{P}\left[L_{s}+S_{s}\right] \\
& =\left(1-\kappa_{21}^{P} \frac{1-e^{-\kappa_{22}^{P}(s-t)}}{\kappa_{22}^{P}}\right) L_{t}+\theta_{2}^{P}+e^{-\kappa_{22}^{P}(s-t)}\left(S_{t}-\theta_{2}^{P}\right)-\kappa_{23}^{P} \frac{e^{-\kappa_{33}^{P}(s-t)}-e^{-\kappa_{22}^{P}(s-t)}}{\kappa_{22}^{P}-\kappa_{33}^{P}}\left(C_{t}-\theta_{3}^{P}\right) .
\end{aligned}
$$


Next, we integrate from $t$ to $t+\tau$ :

$$
\begin{aligned}
\int_{t}^{t+\tau} E_{t}^{P}\left[r_{s}\right] d s= & \int_{t}^{t+\tau}\left(\left[1-\kappa_{21}^{P} \frac{1-e^{-\kappa_{22}^{P}(s-t)}}{\kappa_{22}^{P}}\right] L_{t}+\theta_{2}^{P}+e^{-\kappa_{22}^{P}(s-t)}\left(S_{t}-\theta_{2}^{P}\right)\right. \\
& \left.-\kappa_{23}^{P} \frac{e^{-\kappa_{33}^{P}(s-t)}-e^{-\kappa_{22}^{P}(s-t)}}{\kappa_{22}^{P}-\kappa_{33}^{P}}\left(C_{t}-\theta_{3}^{P}\right)\right) d s \\
= & \theta_{2}^{P} \tau+\left(1-\frac{\kappa_{21}^{P}}{\kappa_{22}^{P}}\right) \tau L_{t}+\frac{\kappa_{21}^{P}}{\kappa_{22}^{P}} L_{t} \int_{t}^{t+\tau} e^{-\kappa_{22}^{P}(s-t)} d s \\
& +\left(S_{t}-\theta_{2}^{P}\right) \int_{t}^{t+\tau} e^{-\kappa_{22}^{P}(s-t)} d s \\
& -\frac{\kappa_{23}^{P}}{\kappa_{22}^{P}-\kappa_{33}^{P}}\left(C_{t}-\theta_{3}^{P}\right) \int_{t}^{t+\tau}\left(e^{-\kappa_{33}^{P}(s-t)}-e^{-\kappa_{22}^{P}(s-t)}\right) d s \\
= & \theta_{2}^{P} \tau+\left(1-\frac{\kappa_{21}^{P}}{\kappa_{22}^{P}}\right) \tau L_{t}-\frac{\kappa_{21}^{P}}{\kappa_{22}^{P}} L_{t}\left[\frac{1}{\kappa_{22}^{P}} e^{-\kappa_{22}^{P}(s-t)}\right]_{t}^{t+\tau} \\
& +\left(S_{t}-\theta_{2}^{P}\right)\left[\frac{-1}{\kappa_{22}^{P}} e^{-\kappa_{22}^{P}(s-t)}\right]_{t}^{t+\tau} \\
& -\frac{\kappa_{23}^{P}}{\kappa_{22}^{P}-\kappa_{33}^{P}}\left(C_{t}-\theta_{3}^{P}\right)\left[\frac{-1}{\kappa_{33}^{P}} e^{-\kappa_{33}^{P}(s-t)}+\frac{1}{\kappa_{22}^{P}} e^{-\kappa_{22}^{P}(s-t)}\right]_{t}^{t+\tau} \\
= & \theta_{2}^{P} \tau+\left(1-\frac{\kappa_{21}^{P}}{\kappa_{22}^{P}}\right) \tau L_{t}+\frac{\kappa_{21}^{P}}{\kappa_{22}^{P}} \frac{1-e^{-\kappa_{22}^{P} \tau}}{\kappa_{22}^{P}} L_{t}+\frac{1}{\kappa_{22}^{P}}\left(S_{t}-\theta_{2}^{P}\right)\left(1-e^{-\kappa_{22}^{P} \tau}\right) \\
& -\frac{\kappa_{23}^{P}}{\kappa_{22}^{P}-\kappa_{33}^{P}}\left(C_{t}-\theta_{3}^{P}\right)\left(\frac{1}{\kappa_{33}^{P}}\left[1-e^{-\kappa_{33}^{P} \tau}\right]-\frac{1}{\kappa_{22}^{P}}\left[1-e^{-\kappa_{22}^{P} \tau}\right]\right) .
\end{aligned}
$$

The relevant term to go into the term premium formula is

$$
\begin{aligned}
\frac{1}{\tau} \int_{t}^{t+\tau} E_{t}^{P}\left[r_{s}\right] d s= & \theta_{2}^{P}+\left(1-\frac{\kappa_{21}^{P}}{\kappa_{22}^{P}}\right) L_{t}+\frac{\kappa_{21}^{P}}{\kappa_{22}^{P}} \frac{1-e^{-\kappa_{22}^{P} \tau}}{\kappa_{22}^{P} \tau} L_{t}+\frac{1-e^{-\kappa_{22}^{P} \tau}}{\kappa_{22}^{P} \tau}\left(S_{t}-\theta_{2}^{P}\right) \\
& -\frac{\kappa_{23}^{P}}{\kappa_{22}^{P}-\kappa_{33}^{P}}\left(\frac{1-e^{-\kappa_{33}^{P} \tau}}{\kappa_{33}^{P} \tau}-\frac{1-e^{-\kappa_{22}^{P} \tau}}{\kappa_{22}^{P} \tau}\right)\left(C_{t}-\theta_{3}^{P}\right) .
\end{aligned}
$$

The final expression for the term premium is given by

$$
\begin{aligned}
T P_{t}(\tau)= & y_{t}(\tau)-\frac{1}{\tau} \int_{t}^{t+\tau} E_{t}^{P}\left[r_{s}\right] d s \\
= & L_{t}+\frac{1-e^{-\lambda \tau}}{\lambda \tau} S_{t}+\left(\frac{1-e^{-\lambda \tau}}{\lambda \tau}-e^{-\lambda \tau}\right) C_{t}-\frac{A(\tau)}{\tau} \\
& -\theta_{2}^{P}-\left(1-\frac{\kappa_{21}^{P}}{\kappa_{22}^{P}}\right) L_{t}-\frac{\kappa_{21}^{P}}{\kappa_{22}^{P}} \frac{1-e^{-\kappa_{22}^{P} \tau}}{\kappa_{22}^{P} \tau} L_{t}-\frac{1-e^{-\kappa_{22}^{P} \tau}}{\kappa_{22}^{P} \tau}\left(S_{t}-\theta_{2}^{P}\right) \\
& +\frac{\kappa_{23}^{P}}{\kappa_{22}^{P}-\kappa_{33}^{P}}\left(\frac{1-e^{-\kappa_{33}^{P} \tau}}{\kappa_{33}^{P} \tau}-\frac{1-e^{-\kappa_{22}^{P} \tau}}{\kappa_{22}^{P} \tau}\right)\left(C_{t}-\theta_{3}^{P}\right) \\
& \frac{\kappa_{21}^{P}}{\kappa_{22}^{P}}\left(1-\frac{1-e^{-\kappa_{22}^{P} \tau}}{\kappa_{22}^{P} \tau}\right) L_{t}+\left(\frac{1-e^{-\lambda \tau}}{\lambda \tau}-\frac{1-e^{-\kappa_{22}^{P} \tau}}{\kappa_{22}^{P} \tau}\right) S_{t} \\
& +\left(\frac{1-e^{-\lambda \tau}}{\lambda \tau}-e^{-\lambda \tau}+\frac{\kappa_{23}^{P}}{\kappa_{22}^{P}-\kappa_{33}^{P}}\left[\frac{1-e^{-\kappa_{33}^{P} \tau}}{\kappa_{33}^{P} \tau}-\frac{1-e^{-\kappa_{22}^{P} \tau}}{\kappa_{22}^{P} \tau}\right]\right) C_{t} \\
& -\left(1-\frac{1-e^{-\kappa_{22}^{P} \tau}}{\kappa_{22}^{P} \tau}\right) \theta_{2}^{P}-\frac{\kappa_{23}^{P}}{\kappa_{22}^{P}-\kappa_{33}^{P}}\left(\frac{1-e^{-\kappa_{33}^{P} \tau}}{\kappa_{33}^{P} \tau}-\frac{1-e^{-\kappa_{22}^{P} \tau}}{\kappa_{22}^{P} \tau}\right) \theta_{3}^{P}-\frac{A(\tau)}{\tau} .
\end{aligned}
$$




\section{References}

Andersen, Torben G. and Luca Benzoni, 2010, "Do Bonds Span Volatility Risk in the U.S. Treasury Market? A Specification Test for Affine Term Structure Models," Journal of Finance, Vol. 65, No. 2, 603-653.

Andersen, Torben G., Tim Bollerslev, Frank X. Diebold, and P. Labys, 2003, "Modeling and Forecasting Realized Volatility," Econometrica, Vol. 71, 579-626.

Bauer, Michael D., Glenn D. Rudebusch, and Jing (Cynthia) Wu, 2012, "Correcting Estimation Bias in Dynamic Term Structure Models," Journal of Business and Economic Statistics, Vol. 30, No. 3, 454-467.

Black, Fisher, 1995, "Interest Rates as Options," Journal of Finance, Vol. 50, No. 7, 1371-1376.

Bomfim, Antulio N., 2003, “'Interest Rates as Options:' Assessing the markets' view of the liquidity trap," Working Paper 2003-45, Finance and Economics Discussion Series, Federal Reserve Board, Washington, D.C.

Christensen, Jens H. E., Francis X. Diebold, and Glenn D. Rudebusch, 2011, "The Affine ArbitrageFree Class of Nelson-Siegel Term Structure Models," Journal of Econometrics, Vol. 164, 4-20.

Christensen, Jens H. E. and Glenn D. Rudebusch, 2012, "The Response of Interest Rates to U.S. and U.K. Quantitative Easing," Economic Journal, Vol. 122, F385-F414.

Christensen, Jens H. E. and Glenn D. Rudebusch, 2013, "Estimating Shadow-Rate Term Structure Models with Near-Zero Yields," Working Paper 2013-07, Federal Reserve Bank of San Francisco.

Collin-Dufresne, Pierre, Robert S. Goldstein, and Chris S. Jones, 2009, "Can Interest Rate Volatility Be Extracted from the Cross-Section of Bond Yields?," Journal of Financial Economics, Vol. 94, 47-66.

Dai, Qiang and Kenneth J. Singleton, 2002, "Expectations Puzzles, Time-Varying Risk Premia, and Affine Models of the Term Structure," Journal of Financial Economics, Vol. 63, 415-441.

Diebold, Francis X. and Glenn D. Rudebusch, 2013, Yield Curve Modeling and Forecasting: The Dynamic Nelson-Siegel Approach, Princeton, NJ: Princeton University Press.

Duffee, Gregory R., 2002, "Term Premia and Interest Rate Forecasts in Affine Models," Journal of Finance, Vol. 57, 405-443.

Duffie, Darrell and Rui Kan, 1996, "A Yield-Factor Model of Interest Rates," Mathematical Finance, Vol. 6, 379-406. 
Fisher, Mark and Christian Gilles, 1996, "Term Premia in Exponential-Affine Models of the Term Structure," Manuscript, Board of Governors of the Federal Reserve System.

Gorovoi, Viatcheslav and Vadim Linetsky, 2004, "Black's Model of Interest Rates as Options, Eigenfunction Expansions and Japanese Interest Rates," Mathematical Finance, Vol. 14, No. 1, 49-78.

Gürkaynak, Refet S., Brian Sack, and Jonathan H. Wright, 2007, "The U.S. Treasury Yield Curve: 1961 to the Present," Journal of Monetary Economics, Vol. 54, 2291-2304.

Ichiue, Hibiki and Yoichi Ueno, 2007, "Equilibrium Interest Rates and the Yield Curve in a Low Interest Rate Environment," Working Paper 2007-E-18, Bank of Japan.

Ichiue, Hibiki and Yoichi Ueno, 2013, "Estimating Term Premia at the Zero Bound: An Analysis of Japanese, US, and UK Yields," Working Paper 2013-E-8, Bank of Japan.

Jacobs, Kris and Lofti Karoui, 2009, "Conditional Volatility in Affine Term Structure Models: Evidence from Treasury and Swap Markets," Journal of Financial Economics, Vol. 91, 288318.

Kim, Don H. and Athanasios Orphanides, 2012, "Term Structure Estimation with Survey Data on Interest Rate Forecasts," Journal of Financial and Quantitative Analysis, Vol. 47, No. 1, 241-272.

Kim, Don H. and Kenneth J. Singleton, 2012, "Term Structure Models and the Zero Bound: An Empirical Investigation of Japanese Yields," Journal of Econometrics, Vol. 170, 32-49.

Kim, Don H. and Jonathan H. Wright, 2005, "An Arbitrage-Free Three-Factor Term Structure Model and the Recent Behavior of Long-Term Yields and Distant-Horizon Forward Rates," Working Paper Finance and Economics Discussion Series 2005-33, Board of Governors of the Federal Reserve System.

Krippner, Leo, 2013, "A Tractable Framework for Zero Lower Bound Gaussian Term Structure Models," Discussion Paper 2013-02, Reserve Bank of New Zealand.

Nelson, Charles R. and Andrew F. Siegel, 1987, "Parsimonious Modeling of Yield Curves," Journal of Business, Vol. 60, 473-489.

Piazzesi, Monika and Eric T. Swanson, 2008, "Futures Prices as Risk-Adjusted Forecasts of Monetary Policy," Journal of Monetary Economics, Vol. 55, 677-691. 
Ueno, Yoichi, Naohiko Baba, and Yuji Sakurai, 2006, "The Use of the Black Model of Interest Rates as Options for Monitoring the JGB Market Expectations," Working Paper 2006-E-15, Bank of Japan. 